\title{
The Milnor Number and Deformations of Complex Curve Singularities
}

\author{
Ragnar-Olaf Buchweitz ${ }^{1 \star}$ and Gert-Martin Greuel ${ }^{2 \star \star}$ \\ ${ }^{1}$ Institut für Mathematik der Universität Hannover, Welfengarten 1, D-3000 Hannover \\ ${ }^{2}$ Mathematisches Institut der Universität Bonn, Wegeler Straße 10, D-5300 Bonn, \\ Federal Republic of Germany
}

\section{Contents}

Introduction

1. The Milnor Number

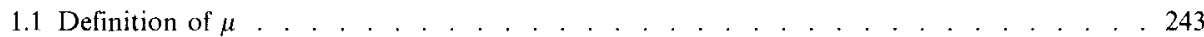

1.2. Some Consequences . . . . . . . . . . . . . . . . . . . . . . . . 245

2. Coherence of the Hypercohomology

2.1. Statement of the Theorem . . . . . . . . . . . . . . . . . . . . . 247

2.2. Proof of the Coherence . . . . . . . . . . . . . . . . . . . . . . . 249

3. Investigation of the Hypercohomology

3.1. A Hypercohomological Gysin Sequence . . . . . . . . . . . . . . . . . . . . . . 251

3.2. Freeness of the Hypercohomology . . . . . . . . . . . . . . . . . . . . . . . . 252

4. Topology of Flat Families of Curves

4.1. Relative Dualizing Sheaf and Normalization . . . . . . . . . . . . . . . . 255

4.2. Milnor Number and Vanishing Cohomology . . . . . . . . . . . . . . . . . . . . 257

5. Topological Equisingularity

5.1. Equisingularity in Higher Codimension . . . . . . . . . . . . . . . . . . . . 260

5.2. Constant Milnor Number is Equivalent to Topological Triviality . . . . . . . . . . . 262

5.3. Comparison to the Case of Plane Curves. . . . . . . . . . . . . . . . . . . . . . 263

6. Other Numerical Invariants

6.1. The Torsion of the Module of Differentials . . . . . . . . . . . . . . . . . . . . 265

6.2. On Zariski's Discriminant Criterion . . . . . . . . . . . . . . . . . . . . . . . . . . 269

7. Examples and Applications

7.1. $\delta$-Constant Deformations . . . . . . . . . . . . . . . . . . . . . 274

7.2. Examples and Counterexamples . . . . . . . . . . . . . . . . . . . . . . . . . . . . 277

References . . . . . . . . . . . . . . . . . . . . . . . . . . . . . . . 280

\section{Introduction}

The main object of this paper is the investigation of a "Milnor number" $\mu$ for an arbitrary reduced complex curve singularity $\left(X_{0}, x_{0}\right) \subset\left(\mathbb{C}^{n}, x_{0}\right)$. The definition, which uses the dualizing module of Grothendieck, is purely local and depends

* Supported by a grant of the DFG

$\star \star$ Supported by a grant of the DFG and partially by the Stiftung Volkswagenwerk at the IHES 
only on the local ring of $\left(X_{0}, x_{0}\right)$. We show that $\mu$ enjoys the following topological properties which are analogous to the classical case of plane curves and which justify the name:

Let $B_{0} \subset \mathbb{C}^{n}$ be a sufficiently small ball with center $x_{0}$ and $X_{0} \subset B_{0}$ a representative of $\left(X_{0}, x_{0}\right)$. Let $D \subset \mathbb{C}$ be a sufficiently small disc with center 0 and $f: X \rightarrow D$ a (flat) deformation of $X_{0}{ }^{1}$. Then $X$ is a complex surface (in general singular) and we may assume that $X$ is contained in $B_{0} \times D$ such that $f$ is the projection to the second factor. Let $\mu\left(X_{t}\right)$ denote the sum over all Milnor numbers of the singular points of $X_{t}=f^{-1}(t)$. We show that $\mu\left(X_{0}\right)-\mu\left(X_{t}\right)$ $=\operatorname{dim}_{\mathbb{C}} H^{1}\left(X_{t}, \mathbb{C}\right.$ ), so $\mu$ measures the vanishing (co-) homology in the family $\left(X_{t}\right)_{t \in D}$ if $t$ tends to 0 . Note that $\mu\left(X_{t}\right)=0$ if and only if $X_{t}$ is smooth, but that there exist curve singularities $\left(X_{0}, x_{0}\right)$ which have no smooth deformations. The other property of $\mu$ is the following. Assume that $\left\{x_{0}\right\} \times D \subset X$ and that $X_{t}$ $-\left\{\left(x_{0}, t\right)\right\}$ is smooth. Then $\mu\left(X_{t},\left(x_{0}, t\right)\right)$ is constant for all $t$ if and only if $f: X \rightarrow D$ is homeomorphic to a product family. These properties of the Milnor number are known for isolated hypersurfaces singularities and were proved by Milnor [Mi] and Lê-Ramanujam [L-R] respectively.

Though we use topological arguments, our methods are mostly algebraic or analytic and make use of the coherence of the hypercohomology of certain complexes. These methods, originally due to Grothendieck, were introduced and used by Brieskorn $[\mathrm{Br}]$ in the study of hypersurface singularities.

Our definition of $\mu$ occurred already implicitly in a paper of Bassein [Ba], who considered only smoothable curves. He introduced the fruitful idea to replace the module of differentials by the dualizing module of Grothendieck.

Here is a short outline of the contents of this paper, each chapter itself being headed by a short summary. The first chapter contains the definition of $\mu$, a proof that it coincides with the classical Milnor number for complete intersection curves, a proof of the formula $\mu=2 \delta-r+1$, which is due to Milnor for plane curves, and some other elementary formulas concerning $\mu$. Chapter 2 and 3 are the most technical parts of the paper, but they are basic for what follows. We give a simple proof, using the main theorem of Kiehl and Verdier $[\mathrm{K}-\mathrm{V}]$, of the coherence theorem mentioned above for families of isolated singularities. This theorem is due to Brieskorn for hypersurfaces and to Hamm in full generality (see also the beginning of 2.1). Moreover we deduce a Gysintype exact sequence and give a criterion for the freeness of the hypercohomology. All this together allows us to give a topological interpretation of $\mu$ in Chap. 4. In Chap. 5 we give a short discussion of (topological) equisingularity in higher codimension (5.1). Note that there is a fundamental difference from the case of plane curves, since the most significant topological invariant of a plane curve singularity, the link, is trivial. Then we state an equisingularity theorem (5.2), part of which was mentioned above. These investigations were initiated by Zariski's "Studies in Equisingularity" $\left[\mathrm{Z}_{1}\right]$ and motivated by the existing vast theory for plane curves (and - more generally - for hypersurfaces). For the readers convenience we give a short summary of these results in 5.3, contrasting them to the general case. The explicit examples and counterexamples are deferred to Chap. 7. In Chap. 6 we use our methods to investigate several other

1 The vague term "sufficiently small" is often used in order not to complicate statements. The precise meaning will become clear from the context and from the proofs. In this case cf. $\$ 2.1$ for a precise definition 
numerical invariants of reduced curve singularities. In particular, we prove a conjecture of Berger concerning the torsion of the module of differentials for curves which have deformations lowering $\delta$ sufficiently. Moreover we apply the coherence theorem to obtain semicontinuity of various numerical invariants. After that we study generic projections and generalize Zariski's discriminant criterion for equisingularity to complete intersection curves. For non-plane curves this criterion is definitely stronger than " $\mu=$ constant". In Chap. 7 we state the announced examples in order to illustrate how the behavior of $\mu$ in general differs from the case of a plane curve. We end with a short discussion of the curve singularity consisting of the $n$ axes in $\mathbb{C}^{n}$. The properties of $\mu$ are already sufficient to determine all its deformations. Some of our results were announced in [B-G].

This work was done while the first author was a guest of the Ecole Polytéchnique at Palaiseau, and the second a guest of the IHES at Bures-sur-Yvette and of the Mathematics Departement of the University of Nice. It is a pleasure to thank these institutions and the mathematicians there for their warm hospitality and support. Especially we would like to thank Lê Dũng Tràng and $\mathrm{B}$. Teissier at the Ecole Polytéchnique, N. A'Campo and P. Deligne at the IHES, J. Briançon, A. Galligo and M. Granger at Nice and last but not least $\mathrm{H}$. Hironaka for fruitful discussions.

We wish to express our thanks to the Deutsche Forschungsgemeinschaft and the Stiftung Volkswagenwerk for financial support.

\section{The Milnor Number}

We define a Milnor number $\mu$ for every arbitrary reduced curve singularity. For the definition we need the notion of a dualizing module in the sense of Grothendieck. More classically this module can be described as the regular differential forms in the sense of Rosenlicht. We show that our definition coincides with the now classical Milnor number of plane curves introduced by Milnor [Mi] and for which the calculation was extended to complete intersection curves in $\left[G_{1}\right]$. From the definition we deduce immediately a formula which relates $\mu$ to the $\delta$-invariant and the number of branches, generalizing a formula of Milnor for plane curves. Finally we characterize the ordinary $n$-tuple points in $\mathbb{C}^{n}$ to be those with minimal $\mu$.

\subsection{Definition of $\mu$}

Let $\left(X_{0}, x_{0}\right) \subset\left(\mathbb{C}^{n}, x_{0}\right)$ be a reduced curve singularity, i.e. the germ of a 1dimensional reduced complex space. Let $\Omega_{X_{0}, x_{0}}^{1}$ be the module of holomorphic 1 -forms on $\left(X_{0}, x_{0}\right)$ (i.e. the module of Kähler differentials) and $\omega_{X_{0}, x_{0}}$ the dualizing module of Grothendieck. $\omega_{X_{0}, x_{0}}$ is uniquely defined only up to non canonical isomorphism; we take the definition of Grothendieck (cf. [A-K], $\left.[\mathrm{Gr}],\left[\mathrm{H}-\mathrm{K}_{1}\right]\right)$ :

$$
\omega_{X_{0}, x_{0}}:=\operatorname{Ext}_{\mathscr{C}_{\mathbb{C}^{n}, x_{0}}^{n-1}}^{n}\left(\mathcal{O}_{X_{0}, x_{0}}, \Omega_{\mathbb{C}^{n}, x_{0}}^{n}\right)
$$

which can be seen to be independant of the embedding $\left(X_{0}, x_{0}\right) \subset\left(\mathbb{C}^{n}, x_{0}\right)$. 
Let $\left.n: \overline{\left(X_{0}, x_{0}\right.}\right) \rightarrow\left(X_{0}, x_{0}\right)$ be the normalization, where $\left(\overline{X_{0}, x_{0}}\right)$ is the multigerm $\left(\bar{X}_{0}, n^{-1}\left(x_{0}\right)\right)$. Using the functoriality of $\Omega^{1}$ and $\omega$ we obtain a mapping

$$
c_{X_{0}}: \Omega_{X_{0}, x_{0}}^{1} \rightarrow n_{*} \Omega_{\overline{\left(\bar{X}_{0}, x_{0}\right)}}^{1} \cong n_{*} \omega_{\left(\overline{\left.X_{0}, x_{0}\right)}\right.} \rightarrow \omega_{X_{0}, x_{0}} .
$$

Composition with the exterior derivation $d$ gives

$$
d: \mathcal{O}_{X_{0}, x_{0}} \stackrel{d}{\longrightarrow} \Omega_{X_{0}, x_{0}}^{1} \stackrel{c_{X_{0}}}{\longrightarrow} \omega_{X_{0}, x_{0}},
$$

which we denote also with $d$.

Definition 1.1.1. Let $\left(X_{0}, x_{0}\right)$ be a reduced curve singularity. We set

$$
\mu=\mu\left(X_{0}, x_{0}\right)=\operatorname{dim}_{\mathbb{C}}\left(\omega_{X_{0}, x_{0}} / d \mathcal{O}_{X_{0}, x_{0}}\right)
$$

and call $\mu$ the Milnor number of $\left(X_{0}, x_{0}\right)$.

We will immediately see that this is a finite number. But first we give another equivalent definition of $\omega_{X_{0}, x_{0}}$. Let $\bar{x}_{0}=n^{-1}\left(x_{0}\right)$ and $\Omega_{\left(\overline{X_{0}}, x_{0}\right)}^{1}\left(\bar{x}_{0}\right)$ be the germs of meromorphic forms on $\left(\overline{X_{0}, x_{0}}\right)$ with a pole (of any order) at most in $\bar{x}_{0}$. We set

$$
\omega_{X_{0}, x_{0}}^{R}=n_{*}\left\{\alpha \in \Omega_{\overline{\left(X_{0}, x_{0}\right)}}^{1}\left(\bar{x}_{0}\right) \mid \sum_{p \in \tilde{x}_{0}} \operatorname{res}_{p}(f \alpha)=0 \quad \forall f \in \mathcal{O}_{X_{0}, x_{0}}\right\} .
$$

These are Rosenlicht's regular differential forms (cf [Se], IV. 9). Again we have a canonical mapping

$$
d: \mathcal{O}_{X_{0}, x_{0}} \rightarrow \Omega_{X_{0}, x_{0}}^{1} \rightarrow n_{*} \Omega_{\left(X_{0}, x_{0}\right)}^{1} \hookrightarrow \omega_{X_{0}, x_{0}}^{R} .
$$

It is proved in $[\mathrm{A}-\mathrm{K}]$, VIII that there is a canonical isomorphism $\omega_{X_{0}, x_{0}} \cong \omega_{X_{0}, x_{0}}^{R}$ such that the following diagram commutes

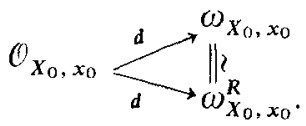

Grothendieck's definition has the advantage that it is obviously functorial and that it can be extended immediately to higher dimensions and to the relative case. On the contrary Rosenlicht's definition is better suited for concrete computations.

Example. $\left(X_{0}, x_{0}\right)=\left(\left\{(x, y, z) \mid x=t^{3}, y=t^{4}, z=t^{5}\right\}, 0\right) \subset\left(\mathbb{C}^{3}, 0\right)$.

$$
d \mathcal{O}_{X_{0}, x_{0}}=t^{2} \mathbb{C}\{t\} d t, \omega_{X_{0}, x_{0}}=\mathbb{C} t^{-3} d t \oplus \mathbb{C} t^{-2} d t \oplus \mathbb{C}\{t\} d t .
$$

Therefore $\mu=4$.

Now we assume that $\left(X_{0}, x_{0}\right)$ is a complete intersection, given as the fibre of the holomorphic mapping $f=\left(f_{1}, \ldots, f_{n-1}\right):\left(\mathbb{C}^{n}, x_{0}\right) \rightarrow\left(\mathbb{C}^{n-1}, 0\right)$. If we intersect the nearby fibre $f^{-1}(t)$ with a sufficiently small ball $B$ centered at $x_{0}$ then $X_{t}$ $=B \cap f^{-1}(t)$ is an open Riemann surface (for sufficiently general, small $t$ ). Its 
first Betti number $b_{1}\left(X_{t}\right)$ is called rank of the vanishing homology or Milnor number of $\left(X_{0}, x_{0}\right)$. Milnor [Mi] showed that for $n=2$

$$
b_{1}\left(X_{t}\right)=\operatorname{dim}_{\mathbb{\mathbb { C }}} \mathcal{O}_{\mathbb{C}^{2}, x_{0}} /\left(\frac{\partial f}{\partial x}, \frac{\partial f}{\partial y}\right) .
$$

For arbitrary complete intersection curves we have

$$
b_{1}\left(X_{t}\right)=\operatorname{dim}_{\mathbb{C}} \Omega_{X_{0}, x_{0}}^{1} / d \mathcal{O}_{X_{0}, x_{0}} .
$$

This was proved in $\left[\mathrm{G}_{1}\right]$.

The interpretation of $\mu$ as rank of the vanishing homology in general will be discussed in Chap. 4. Here we give a direct algebraic argument which shows the coincidence in the case of a complete intersection (cf. also Corollary 6.1.6).

Lemma 1.1.2. Let $\left(X_{0}, x_{0}\right)$ be a reduced complete intersection curve, then

$$
\mu=\operatorname{dim}_{\mathbb{C}} \Omega_{X_{0}, x_{0}}^{1} / d \Theta_{X_{0}, x_{0}} .
$$

Proof. For complete intersections the dualizing module is equal to

$$
\omega_{X_{0}, x_{0}}=\left(\frac{d x_{1} \wedge \ldots \wedge d x_{n}}{d f}\right) \mathcal{O}_{X_{0}, x_{0}}
$$

(cf. [Se]) where we have written $d f$ instead of $d f_{1} \wedge \ldots \wedge d f_{n-1}$. Exterior multiplication with $d f$ induces an isomorphism

$$
\omega_{X_{0}, x_{0}} / d \mathcal{O}_{X_{0}, x_{0}} \stackrel{\wedge d f}{\cong} \Omega_{\mathbb{C}^{n}, x_{0}}^{n} \otimes \mathcal{O}_{X_{0}, x_{0}} / d f \wedge d \mathcal{O}_{X_{0}, x_{0}} .
$$

Now consider the exact sequence

$$
\begin{aligned}
0 & \rightarrow T \Omega_{X_{0}, x_{0}}^{1} \rightarrow \Omega_{X_{0}, x_{0}}^{1} / d \mathcal{O}_{X_{0}, x_{0}} \stackrel{\wedge d f}{\longrightarrow} \Omega_{\mathbb{C}^{n}, x_{0}}^{n} \otimes \mathcal{O}_{X_{0}, x_{0}} / d f \wedge d \mathcal{O}_{X_{0}, x_{0}} \\
& \rightarrow \Omega_{\mathbb{C}^{n}, x_{0}}^{n} \otimes \mathcal{O}_{X_{0}, x_{0}} / d f \wedge \Omega_{X_{0}, x_{0}}^{1} \rightarrow 0
\end{aligned}
$$

where $T \Omega_{X_{0}, x_{0}}^{1}$ is the torsion submodule of $\Omega_{X_{0}, x_{0}}^{1}$.

But it was shown in $\left[G_{1}\right]$, Proposition 1.11 iii) that

$$
\operatorname{dim}_{\mathbb{C}} T \Omega_{X_{0}, x_{0}}^{1}=\operatorname{dim}_{\mathbb{C}} \Omega_{\mathbb{C}^{n}, x_{0}}^{n} \otimes \mathcal{O}_{X_{0}, x_{0}} / d f \wedge \Omega_{X_{0}, x_{0}}^{1},
$$

which proves our assertion.

\subsection{Some Consequences}

Let $\delta=\delta\left(X_{0}, x_{0}\right)=\operatorname{dim}_{\mathbb{C}} n_{*} \mathcal{O}_{\left(\overline{X_{0}, x_{0}}\right)} / \mathcal{O}_{X_{0}, x_{0}}$ be the $\delta$-invariant and $r=r\left(X_{0}, x_{0}\right)$ the number of irreducible components of $\left(X_{0}, x_{0}\right)$. The following lemma is due to 
Milnor [Mi] for plane curves; his proof was generalized to complete intersections by Giusti [Mi]. The following proof is due to Bassein [Ba], who considered smoothable curves. From our point of view Milnor's formula is a theorem about residues and duality.

Proposition 1.2.1. 1) $\mu=2 \delta-r+1$,

2) $\mu=0$ iff $\left(X_{0}, x_{0}\right)$ is nonsingular.

Proof. Consider the exact sequences

$$
\begin{aligned}
& 0 \rightarrow \mathbb{C}^{r-1} \rightarrow n_{*} \mathcal{O}_{\left(\overline{X_{0}, x_{0}}\right)} / \mathcal{O}_{X_{0}, x_{0}} \stackrel{d}{\rightarrow} n_{*} \Omega_{\left(\overline{X_{0}}, x_{0}\right)}^{1} / d \mathcal{O}_{X_{0}, x_{0}} \rightarrow 0, \\
& 0 \rightarrow n_{*} \Omega_{\left(\overline{\left.X_{0}, x_{0}\right)}\right.}^{1} / d \mathcal{O}_{X_{0}, x_{0}}^{\rightarrow} \omega_{X_{0}, x_{0}} / d \mathcal{O}_{X_{0}, x_{0}} \rightarrow \omega_{X_{0}, x_{0}} / n_{*} \Omega_{\left(\overline{X_{0}}, x_{0}\right)}^{1} \rightarrow 0 .
\end{aligned}
$$

Using Rosenlicht's definition we see that there is a perfect pairing (cf. [Se], IV.9)

$$
\left(n_{*} \mathcal{O}_{\overline{\left.X_{0}, x_{0}\right)}} / \mathcal{O}_{X_{0}, x_{0}}\right) \times\left(w_{X_{0}, x_{0}} / n_{*} \Omega_{\left(\overline{X_{0}, x_{0}}\right)}^{1}\right) \rightarrow \mathbb{C},
$$

given by $[f] \times[\alpha] \mapsto \sum_{p \in \bar{x}_{0}} \operatorname{res}_{p}(f \alpha)$. So both modules have length $\delta$ and 1) follows.

2) is now an easy consequence.

Assume $\left(X_{0}, x_{0}\right)=\bigcup_{i=1}^{r}\left(X_{0}^{i}, x_{0}\right)$ such that $X_{0}^{i} \cap X_{0}^{j}=\left\{x_{0}\right\}$ for $i \neq j\left(\left(X_{0}^{i}, x_{0}\right)\right.$ need not be irreducible). Set

$$
\left(X_{i}, x_{0}\right)=\bigcup_{j=i+1}^{r}\left(X_{0}^{j}, x_{0}\right)
$$

$\delta_{i}=\delta\left(X_{0}^{i}, x_{0}\right)$ and $\mu_{i}=\mu\left(X_{0}^{i}, x_{0}\right)$. For two arbitrary curves $\left(C_{i}, x_{0}\right) \subset\left(\mathbb{C}^{n}, x_{0}\right)$, $i=1,2$, we set

$$
\left(C_{1} \cdot C_{2}\right)=\operatorname{dim}_{\mathbb{C}} \mathcal{O}_{\mathbb{C}^{n}, x_{0}} / I_{1}+I_{2}
$$

where $I_{i}$ is the ideal of $\left(C_{i}, x_{0}\right)$ in $\mathscr{O}_{\mathbb{C}^{n}, x_{0}}$.

Lemma 1.2.2. (Hironaka [Hi]). $\delta=\sum_{i=1}^{r} \delta_{i}+\sum_{i=1}^{r-1}\left(X_{0}^{i} \cdot X_{i}\right)$

Proof. We may assume $r=2$, the general case follows by induction. Let $\mathscr{O}$ $=\mathcal{O}_{\mathbb{C}^{n}, x_{0}}, I=I_{1} \cap I_{2}$ the ideal of $\left(X_{0}, x_{0}\right) \subset\left(\mathbb{C}^{n}, x_{0}\right)$. Consider the inclusions

$$
\mathscr{O} / I \hookrightarrow \mathcal{O} / I_{1} \oplus \mathcal{O} / I_{2} \hookrightarrow \overline{\mathcal{O} / I_{1}} \oplus \overline{\mathcal{O} / I_{2}}=\mathcal{O}_{\left(\overline{\left.X_{0}, x_{0}\right)}\right.},
$$

where $\overline{\mathcal{O} / I_{i}}$ denotes the integral closure in its total ring of fractions. Now the lemma follows from the exact sequence

$$
0 \rightarrow \mathcal{O} / I \rightarrow \mathcal{O} / I_{1} \oplus \mathcal{O} / I_{2} \rightarrow \mathcal{O} / I_{1}+I_{2} \rightarrow 0 .
$$

With the same notations we obtain

Corollary 1.2.3.1) $\mu-1=\sum_{i=1}^{r}\left(\mu_{i}-1\right)+2 \sum_{i=1}^{r-1}\left(X_{0}^{i} \cdot X_{i}\right)$.

2) $\mu \geqq \sum_{i=1}^{r} \mu_{i}+r-1$. 
Remark. For a plane curve $\left(X_{0}, x_{0}\right)$ the number $\left(X_{0}^{i} \cdot X_{i}\right)$ is the intersection multiplicity. In particular $\left(X_{0}^{1} \cdot\left(X_{0}^{2} \cup X_{0}^{3}\right)\right)=\left(X_{0}^{1} \cdot X_{0}^{2}\right)+\left(X_{0}^{1} \cdot X_{0}^{3}\right)$. This is not true in general as can be seen already from the following example.

Example. Let $\left(X_{0}, x_{0}\right) \subset\left(\mathbb{C}^{n}, x_{0}\right)$ be the union of the $n$ coordinate axes, which is defined by $x_{i} x_{j}=0,1 \leqq i<j \leqq n$. Since $\left(X_{0}^{i} \cdot X_{i}\right)=1$ we obtain $\mu=n-1$. We call each singularity analytically equivalent to $\left(X_{0}, x_{0}\right)$ the ordinary n-tuple point. It can be characterized by the minimality of $\mu$ :

Lemma 1.2.4. Let $\left(X_{0}, x_{0}\right)$ be a reduced curve singularity. Then

i) $\mu \geqq \delta$.

ii) If $\left(X_{0}, x_{0}\right) \subset\left(\mathbb{C}^{n}, x_{0}\right)$ is minimally embedded then $\mu \geqq n-1$.

iii) If one of the foregoing inequalities is an equality then also the other one and $\left(X_{0}, x_{0}\right)$ is the ordinary n-tuple point.

Proof. Since $\delta \geqq r-1$ and $\delta=r-1$ iff $\left(X_{0}, x_{0}\right)$ is the ordinary $n$-tuple point, i) and one half of iii) follow from Proposition 1.2.1.

Let $m$ be the multiplicity of $\left(X_{0}, x_{0}\right)$. It is well known that for a curve $m \geqq n$ where $n$ is the embedding dimension. We have also $\delta \geqq m-1$ by

$$
\delta=\operatorname{dim}_{\mathbb{C}}\left(n_{*} \mathcal{O}_{\overline{X_{0}, x_{0}}} / \mathfrak{M}\right)-1 \geqq \operatorname{dim}_{\mathbb{C}}\left(n_{*} \mathcal{O}_{\overline{X_{0}, x_{0}}} / \mathfrak{M} n_{*} \mathcal{O}_{X_{0}, x_{0}}\right)-1=m-1
$$

$\left(\mathfrak{M}\right.$ denotes the maximal ideal of $\mathcal{O}_{X_{0}, x_{0}}$ ).

Hence

$$
\mu=2 \delta-r+1 \geqq(m-1)+\delta-r+1 \geqq n-1
$$

and equality holds iff $\delta=r-1$.

\section{Coherence of the Hypercohomology}

In this section we consider flat families $f: X \rightarrow D$ of complex spaces of arbitrary dimension with at most isolated singularities, where the parameter-space is a small disc $D \subset \mathbb{C}$. We consider rather general complexes $\left(\mathscr{K}^{*}, d\right)$ of sheaves on $X$ of which we are going to prove the coherence of the hypercohomology with respect to the direct image functor $f_{*}$. This generality is needed in order to cover all our applications. A good example one should keep in mind is the complex $\left(\Omega_{X / D}^{*}, d\right)$ of relative differential forms of $X / D$, although later modified versions of this complex are more important.

The coherence theorem and the Gysin sequence of Sect. 3 are the essential tools for our investigation of $\mu$ and other numerical invariants in flat families of curves. These tools were introduced by Grothendieck and used by Deligne, Katz and others for the study of proper smooth families of algebraic varieties. They were developped and modified by Brieskorn $[\mathrm{Br}]$ in order to investigate algebraically the topological invariants of isolated hypersurface singularities. Generalizations to complete intersections were carried out in $\left[\mathrm{G}_{1}\right]$ and later by Hamm [Ha] to arbitrary singularities.

\subsection{Statement of the Theorem}

The coherence for $\Omega_{X / D}^{*}$ is already known and was proved by Hamm [Ha] under somewhat more general conditions. Hamm's proof, which has not been pub- 
lished, is rather complicated due to the fact that he treats also non isolated singularities and therefore has to use Hironaka's resolution of singularities. So we decided to present our simple proof for all $\left(\mathscr{K}^{*}, d\right)$. Using a Mayer-Vietoris argument of Brieskorn's proof for hypersurfaces $[\mathrm{Br}]$ we show that the coherence is an easy consequence from the main theorem of Kiehl-Verdier [K-V]. Though we are later only interested in the case of curves we treat here the general case since it is not more complicated.

Let $\left(X_{0}, x_{0}\right)$ be the germ of a pure $m$-dimensional complex space $(m \geqq 1)$ with isolated singularity, let $D \subset \mathbb{C}$ be a small open disc with center 0 and $f:\left(X, x_{0}\right) \rightarrow(D, 0)$ a (flat) deformation of $\left(X_{0}, x_{0}\right)$. This implies that $\left(X, x_{0}\right)$ is pure $(m+1)$-dimensional and $\left(f^{-1}(0), x_{0}\right)=\left(X_{0}, x_{0}\right)$.

Once and for all we choose a "good" representative for $f$ : Let $X_{0}$ be embedded in a small open ball $B_{0} \subset \mathbb{C}^{n}$ with center $x_{0}$. We may assume that $X$ is a closed analytic subset of $B=B_{0} \times D$ and $f: X \rightarrow D$ is the restriction of the projection on $D . C(f) \subset X$ denotes the set of critical points of $f$. We shall identify $B_{0}$ with $B_{0} \times\{0\}$.

If $B_{0}$ is sufficiently small and $D$ is sufficiently small with respect to $B_{0}$ we may assume that the following holds:

a) $X$ and $X_{0}$ are contractible and $X_{0}-\left\{x_{0}\right\}$ is nonsingular,

b) $f: X \rightarrow D$ is flat and $\left.f\right|_{C(f)}: C(f) \rightarrow D$ is finite,

c) $\partial B_{0} \times\{t\}$ intersects $X_{t}=f^{-1}(t)$ transversally in regular points of $X_{t}$ for all $t \in D$ and each sphere $S_{\varepsilon}^{2 n-1} \subset B_{0}$ with center $x_{0}$ intersects $X_{0}$ transversally.

Under these assumptions $\operatorname{dim} C(f) \leqq 1$ holds and all fibres $X_{t}, t \in D$, are $n$ dimensional Stein complex spaces with at most isolated singularities.

We define now a rather general class of complexes and study their hypercohomology.

Let $\left(\mathscr{K}^{\circ}, d\right)$ be a finite complex of sheaves on $X$,

$$
\mathscr{K}^{*}: 0 \rightarrow \mathscr{K}^{0} \stackrel{d^{0}}{\longrightarrow} \mathscr{K}^{1} \stackrel{d^{1}}{\longrightarrow} \mathscr{K}^{2} \rightarrow \ldots
$$

with the following properties:

$\left(P_{1}\right) \mathscr{K}^{p}$ is a coherent $\mathcal{O}_{X}$-module for all $p$,

$\left(P_{2}\right) \quad d^{p}$ is $f^{-1} \mathcal{O}_{D}$-linear for all $p$,

$\left(P_{3}\right) \quad \mathscr{H}^{0}\left(\mathscr{K}^{*}\right)=f^{-1} \mathscr{F}$ where $\mathscr{F}$ is an $\mathcal{O}_{D^{-}}$module,

$\left.\left(P_{4}\right) \quad \mathscr{K}^{p}\right|_{X-C(f)}$ is a free $\mathscr{O}_{X-C(f)}$-module $(p \geqq 0)$ and $\left(\left.\mathscr{K}^{*}\right|_{X-C(f)},\left.d^{*}\right|_{X-C(f)}\right)$ is exact for $p>0$.

$\left(f^{-1} \mathscr{F}\right.$ denotes the topological preimage sheaf).

The sheaves of hypercohomology $\mathbb{R}^{p} f_{*}\left(\mathscr{K}^{*}\right)$ (cf. [EGA]) are $\mathcal{O}_{D^{-} \text {-modules and }}$ the aim of this section is the following theorem.

Theorem 2.1.1. (Brieskorn, Hamm).

(1) $\mathbb{R}^{p} f_{*}\left(\mathscr{K}^{\circ}\right)$ is a coherent $\mathcal{O}_{D^{-}}$-module for all $p$,

(2) $\mathbb{R}^{p} f_{*}\left(\mathscr{K}^{*}\right) \cong \mathscr{H}^{p}\left(f_{*} \mathscr{K}^{*}\right)$,

(3) $\left(\mathbb{R}^{p} f_{*}\left(\mathscr{K}^{*}\right)\right)_{0} \cong \mathscr{H}^{p}\left(\mathscr{K}_{x_{0}}^{*}\right)$.

$\left(\mathscr{F}_{x}\right.$ denotes the stalk of the sheaf $\mathscr{F}$ in $\left.x\right)$. 
This theorem (for $\left(\mathscr{K}^{*}, d\right)=\left(\Omega_{X / D}^{*}, d\right)$ ) was proved by Brieskorn [Br] for hypersurfaces and Hamm [Ha] in the general case.

Remark. The fact that $\left.\mathscr{K}^{p}\right|_{X-C(f)}$ is free, is used only in 3.2.2-3.2.4.

\subsection{Proof of the Coherence}

For the proof of Theorem 2.1.1 we need some preparations.

Proposition 2.2.1. Let $B_{0}^{\prime} \subset B_{0}$ be an open ball with center $x_{0}$, let $\varepsilon$ resp. $\varepsilon^{\prime}$ be the radii of $B_{0}$ resp. $B_{0}^{\prime}$ and assume $0<\left|\varepsilon-\varepsilon^{\prime}\right|$ to be sufficiently small. Then for each open subset $\mathscr{U} \subset D$ and for each $p$ the restriction maps induce isomorphisms

$$
\begin{aligned}
H^{p}\left(f^{-1}(\mathscr{U}), f^{-1} \mathscr{F}\right) & \cong H^{p}\left(f^{-1}(\mathscr{U}) \cap \bar{B}_{0}^{\prime} \times D, f^{-1} \mathscr{F}\right) \\
& \cong H^{p}\left(f^{-1}(\mathscr{U}) \cap B_{0}^{\prime} \times D, f^{-1} \mathscr{F}\right) .
\end{aligned}
$$

Proof. Because of the assumption 2.1 (c) it is possible to find a vector field in the neighbourhood of $X-X \cap B_{0}^{\prime} \times D$ which respects the fibers of $f$ such that the integral curves induce a strong deformation retraction from $X$ onto $X \cap B_{0}^{\prime} \times D$. Using a Mayer-Vietoris sequence the proposition follows as in Brieskorn [Br], p. $20,21$.

Corollary 2.2.2. $R^{p} f_{*}\left(f^{-1} \tilde{\mathscr{F}}\right)_{t} \cong H^{p}\left(X_{t}, \mathscr{F}\right), t \in D$.

Proof. This follows from [Go], Theorem 4.11.1.

Lemma 2.2.3. With the notations of 2.1 and Propositions 2.2.1 the following holds: For each Stein open subset $\mathscr{U} \subset D$ the restriction map

$$
\Gamma\left(f^{-1}(\mathscr{U}), \mathscr{K}^{*}\right) \rightarrow \Gamma\left(f^{-1}(\mathscr{U}) \cap B_{0}^{\prime} \times D, \mathscr{K}^{*}\right)
$$

is a quasi-isomorphism (i.e. induces an isomorphism of the cohomology groups).

Proof. Consider the second spectral sequence of the hypercohomology of the functor $\Gamma$ with respect to $\mathscr{K}^{*}$. The restriction map induces the following commutative diagram:

$$
\begin{aligned}
& E_{2}^{p q}=H^{p}\left(f^{-1}(\mathscr{U}), \mathscr{H}^{q}\left(\mathscr{K}^{*}\right)\right) \quad \Rightarrow \mathbb{H}^{p+q}\left(f^{-1}(\mathscr{U}), \mathscr{K}^{*}\right) \\
& E_{2}^{p q}=H^{p}\left(f^{-1}(\mathscr{U}) \cap B_{0}^{\prime} \times D, \mathscr{H} \mathscr{H}^{q}(\mathscr{K})\right) \Rightarrow \mathbb{H}^{p+q}\left(f^{-1}(\mathscr{U}) \cap B_{0}^{\prime} \times D, \mathscr{K}^{*}\right) .
\end{aligned}
$$

$E_{2}^{p q} \rightarrow E_{2}^{p q}$ is an isomorphism for $q>0$ if $C(f) \cap\left(X-X \cap B_{0}^{\prime} \times D\right)=\emptyset$, which is true for $\left|\varepsilon-\varepsilon^{\prime}\right|$ sufficiently small. The isomorphism $E_{2}^{p 0} \rightarrow{ }^{\prime} E_{2}^{p 0}$ follows from Proposition 2.2.1. because of property $2.1\left(\mathrm{P}_{3}\right)$. Therefore the mapping between the hypercohomology groups is an isomorphism. On the other hand the first spectral sequence

$$
H^{p}\left(H^{q}\left(f^{-1}(\mathscr{U}), \mathscr{K}^{*}\right)\right) \Rightarrow \mathbb{I H}^{p+q}\left(f^{-1}(\mathscr{U}), \mathscr{K}^{*}\right)
$$

shows that $H^{p}\left(\Gamma\left(f^{-1}(\mathscr{U}), \mathscr{K}^{\bullet}\right)\right) \cong \mathbb{H}^{p}\left(f^{-1}(\mathscr{U}), \mathscr{K}^{\bullet}\right)$ since $f^{-1}(\mathscr{U})$ is Stein and $\mathscr{K}^{p}$ is coherent. The same holds over $f^{-1}(\mathscr{U}) \cap B_{0}^{\prime} \times D$ and the lemma is proved. 
We are now in the position to apply the main theorem of Kiehl and Verdier ([K-V], Theorem 3.7).

Theorem 2.2.4. (Kiehl-Verdier). Let $\left(A_{i}\right)_{t \in[0,1]}$ be a nuclear chain of Fréchet algebras and let $N^{*}, M^{*}$ be finite complexes of nuclear $A_{0}$-modules. Moreover let $\varphi^{*}: N^{*} \rightarrow M^{*}$ be an $A_{0}$-linear subnuclear quasiisomorphism of complexes and let $A_{t}$ be transversal (over $A_{0}$ ) to $N^{p}, M^{p}$ for all $p$ and all $t$. Then there exists a finite complex $F^{*}$ of free, finitely generated $A_{1}$-modules and a quasiisomorphism $F^{*} \rightarrow A_{1} \hat{\otimes}_{A_{0}} N^{*}$.

Proof of Theorem 2.1.1. Let $D=D_{\delta}$ be the disc in $\mathbb{C}$ of radius $\delta$ with center $0 \in \mathbb{C}$ and let $0<\rho<\delta$. We put

$$
\begin{aligned}
A_{t} & =\Gamma\left(D_{\delta-t \rho}, \mathcal{O}_{D}\right), \\
N^{*} & =\Gamma\left(X, \mathscr{K}^{*}\right)=\Gamma\left(D, f_{*} \mathscr{K}^{*}\right), \\
M^{*} & =\Gamma\left(X \cap B_{0}^{\prime} \times D, \mathscr{K}^{*}\right)=\Gamma\left(D,\left(f \mid X \cap B_{0}^{\prime} \times D\right)_{*} \mathscr{K}^{*}\right), \\
\varphi^{\bullet} & : N^{*} \rightarrow M^{*} \text { the restriction map. }
\end{aligned}
$$

By Lemma 2.2.2 $\varphi^{\circ}$ is a quasiisomorphism, and this is indeed the reason for the coherence. All other assumptions in the theorem of Kiehl-Verdier are of general nature and they are fullfilled since $f$ is a Stein mapping of complex spaces. We refer to [Do], where all the properties we need are stated. By the theorem there exists a complex $F^{*}$ with $F^{p} \cong A_{1}^{k_{p}}$ for suitable $k_{p}$ and a quasiisomorphism

$$
h: F^{*} \rightarrow \Gamma\left(D_{\delta-\rho}, f_{*} \mathscr{K}^{*}\right) .
$$

Let $\mathscr{F}^{p}=\mathcal{O}_{D_{\delta-\rho}}^{k_{p}}$ and $\mathscr{F}^{*}$ the sheaf complex associated to $F^{*}$. For each Stein open subset $\mathscr{U} \subset D_{\delta-\rho} h$ induces an isomorphism

$$
\Gamma\left(\mathscr{U}, \mathscr{H}^{p}\left(\mathscr{F}^{*}\right)\right) \stackrel{\cong}{\longrightarrow} H^{p}\left(\Gamma\left(f^{-1}(\mathscr{U}), \mathscr{K}^{\bullet}\right)\right)
$$

(cf. [Do], Prop. 2 and Cor. to Prop. 3). Therefore

$$
\mathscr{H}^{p}\left(f_{*} \mathscr{K}^{*}\right) \cong \mathscr{H}^{p}\left(\mathscr{F}^{*}\right)
$$

and the second sheaf is coherent. Since $f$ is Stein, the spectral sequence $\mathscr{H}^{p}\left(R^{q} f_{*} \mathscr{K}^{*}\right) \Rightarrow \mathbb{R}^{p+q} f_{*}\left(\mathscr{K}^{*}\right)$ degenerates and therefore $\mathscr{H}^{p}\left(f_{*}\left(\mathscr{K}^{*}\right)\right) \cong \mathbb{R}^{p} f_{*}\left(\mathscr{K}^{*}\right)$. This proves 2.1.1 (1) and (2).

2.1.1. (3) results from the fact that $X_{0}$ is contractible (cf. $\left[G_{1}\right]$, Prop. 3.1. and the proof of Prop. 3.1.1).

\section{Investigation of the Hypercohomology}

We keep all the notations and assumptions of 2.1, but from now on we specialize to families of curves, i.e. we suppose $m=1$.

The reason why we restrict our investigations to families of curves (at least for the moment) becomes clear in this section: Stein spaces of dimension 1 have nonvanishing cohomology groups only in degree 0 and 1 , in particular they have spherical fibre cohomology. It follows from the Leray spectral sequence that for 
fibrations with spherical fiber there exists a long exact sequence, the "Gysin sequence". We show an analogous result for the hypercohomology in 3.1, though the actual role of the base and the fiber is just opposite to the case of the Leray spectral sequence. In 3.2 we deduce necessary and sufficient conditions for the hypercohomology to be free. Moreover we determine the difference between the rank and the minimal number of generators of the hypercohomology, a result which will be used in Chap. 6 for the computation resp. comparison of several analytic invariants of curves. The principle how we are going to use the Gysin sequence and the freeness is explained at the end of 3.1 and 3.2 respectively.

\subsection{A Hypercohomological Gysin Sequence}

Let $f: X \rightarrow D$ be a good representative of a flat family $f:\left(X, x_{0}\right) \rightarrow(D, 0)$ of reduced curves and let $\mathscr{K}^{\circ}$ be a complex which satisfies $\left(P_{1}\right), \ldots,\left(P_{4}\right)(\mathrm{cf} .2 .1)$.

Proposition 3.1.1. Let $t \in D$ and $S\left(X_{t}\right)$ be the (finite) set of singular points of $X_{t}$. The following holds:

(1) $\mathbb{R}^{0} f_{*}\left(\mathscr{K}^{*}\right)_{t} \cong H^{0}\left(X_{t}, \mathbb{C}\right) \otimes_{\mathbb{C}} \mathscr{F}_{t}$

(2) $\mathbb{R}^{p} f_{*}\left(\mathscr{K}^{*}\right)_{t} \cong \bigoplus_{x \in S\left(X_{t}\right)} H^{p}\left(\mathscr{K}_{x}^{*}\right), \quad p>1$,

(3) There is a canonical exact sequence, the "Gysin sequence"

$$
0 \rightarrow H^{1}\left(X_{t}, \mathbb{C}\right) \otimes_{\mathbb{C}} \mathscr{F}_{t} \rightarrow \mathbb{R}^{1} f_{*}\left(\mathscr{K}^{*}\right)_{t} \rightarrow \underset{x \in S\left(X_{t}\right)}{\oplus} H^{1}\left(\mathscr{K}_{x}^{*}\right) \rightarrow 0 .
$$

Remark. The proof makes use only of the properties $\left(\mathrm{P}_{3}\right)$ and $\left(\mathrm{P}_{4}\right)$ of 2.1 but not of the coherence.

Proof. Consider the second spectral sequence

$$
E_{2}^{p q}=R^{p} f_{*}\left(\mathscr{H}^{q}\left(\mathscr{K}^{*}\right)\right)_{t} \Rightarrow \mathbb{R}^{p+q} f_{*}\left(\mathscr{K}^{\bullet}\right)_{t} .
$$

Let $q>0 . \mathscr{H}^{q}\left(\mathscr{K}^{\circ}\right)$ is concentrated on $C(f)$ and $\left.f\right|_{C(f)}$ is proper, therefore

$$
E_{2}^{p q} \cong H^{p}\left(X_{t}, \mathscr{H}^{q}\left(\mathscr{K}^{*}\right)\right)
$$

Because of Corollary 2.2.2 this isomorphism holds also for $q=0$. From the universal coefficient theorem we deduce

$$
\text { (*) } \quad E_{2}^{p 0} \cong H^{p}\left(X_{1}, \mathbb{C}\right) \otimes_{\mathbb{C}} \mathscr{F}_{i} .
$$

But $X_{t}$ is Stein and 1-dimensional. Therefore $E_{2}^{p 0}=0$ for $p \neq 0$, 1 . Since for $q \neq 0$ $\mathscr{H}^{q}\left(\mathscr{K}^{\circ}\right) \mid X_{t}$ is concentrated on finitely many points by $\left(\mathrm{P}_{4}\right)$ of 2.1 we obtain $E_{2}^{p q}$ $=0$ for $p \neq 0, q \neq 0$ and $E_{2}^{0 q} \cong \underset{x \in S\left(X_{t}\right)}{\oplus} H^{q}\left(\mathscr{K}_{x}^{*}\right)$. Hence we obtain an exact sequence which we call (hypercohomological) Gysin sequence (cf. also [Go], 4.5.1, 4.6.1),

$$
\ldots \rightarrow E_{2}^{1, p-1} \rightarrow \mathbb{R}^{p} f_{*}\left(\mathscr{K}^{n}\right)_{t} \rightarrow E_{2}^{0 p} \rightarrow E_{2}^{1 p} \rightarrow \ldots
$$

Therefore (1) results from (*), and (2), (3) from the Gysin sequence. 
The Gysin sequence shows that the hypercohomology consists of two terms, a topological and more global one (namely $\left.H^{1}\left(X_{t}, \mathbb{C}\right) \otimes \mathscr{F}_{t}\right)$ and a local analytical part which is concentrated in the singular points of the fibers.

Our method how we use the Gysin sequence several times is the following: Let $\alpha\left(X_{0}, x_{0}\right)$ be any analytical invariant of $\left(X_{0}, x_{0}\right)$ such that $\alpha\left(X_{0}, x_{0}\right)=0$ if $x_{0}$ is a smooth point on $X_{0}$. Assume there exists a complex $\mathscr{K}^{\circ}$ on $X$ as above such that $\alpha\left(X_{0}, x_{0}\right)=\operatorname{dim}_{\mathbb{C}} H^{1}\left(\mathscr{K}_{x_{0}}^{*}\right) / f \cdot H^{1}\left(\mathscr{K}_{x_{0}}^{*}\right)$, and for simplicity suppose $\mathscr{F}=\mathscr{C}_{D}$. Consider now the Gysin sequence for $t=0$ and $t \neq 0$. The coherence theorem implies

$$
\alpha\left(X_{0}, x_{0}\right) \geqq \sum_{x \in X_{\mathfrak{t}}} \alpha\left(X_{t}, x\right)+\operatorname{dim}_{\mathbb{C}} H^{1}\left(X_{t}, \mathbb{C}\right) .
$$

If $\mathbb{R}^{1} f_{*}\left(\mathscr{K}^{*}\right)$ is free we even have equality. This will be the case for $\alpha=\mu$. Of course, not every analytic invariant of $\left(X_{0}, x_{0}\right)$ can be sheafified in this way.

Therefore it is useful to look for conditions on $\mathscr{K}^{*}$ which guarantee that $\mathbb{R}^{1} f_{*}\left(\mathscr{K}^{\circ}\right)$ is free. More generally we are interested in the missing term in the above inequality. These problems will be considered in the following section.

\subsection{Freeness of the Hypercohomology}

Consider the following condition on the complex $\left(\mathscr{K}^{*}, d\right)$ :

$\left(\mathrm{P}_{5}\right)$ The canonical mapping $\mathscr{H}^{0}\left(\mathscr{K}^{0}\right) / f \mathscr{H}^{0}\left(\mathscr{H}^{\circ}\right) \rightarrow \mathscr{H}^{0}\left(\mathscr{K}^{\circ} / f \mathscr{K}^{\circ}\right)$ is bijective.

Since $d: \mathscr{K}^{*} \rightarrow \mathscr{K}^{*+1}$ is $f^{-1} \mathcal{O}_{D^{-}}$-linear it induces a $\mathbb{C}$-linear mapping $d_{X_{0}}$ on $\mathscr{K}_{X_{0}}^{*}=\mathscr{K}^{*} / f \mathscr{K}^{*} \mid X_{0}$.

Remark. The assumption $\left(\mathrm{P}_{5}\right)$ implies that the complex $\left(\mathscr{K}_{X_{0}}^{*}, d_{X_{0}}\right)$ is almost an "absolute version" of our complex $\left(\mathscr{K}^{\prime}, d\right)$. This means the following: $\left(\mathscr{K}_{X_{0}}, d_{X_{0}}\right)$ satisfies all the properties $\left(\mathrm{P}_{1}\right), \ldots,\left(\mathrm{P}_{4}\right)$ with respect to the mapping $f: X_{0} \rightarrow\{0\}$, except perhaps the exactness property $\left(\mathrm{P}_{4}\right)$. If $\mathscr{K}_{x_{0}}^{*}$ has this property, i.e. $\left(\left.\mathscr{K}_{X_{0}}\right|_{X_{0}-\left\{x_{0}\right\}},\left.d_{X_{0}}\right|_{X_{0-\left\{x_{0}\right\}}}\right)$ is exact for $p>0$, then all the statements of $\S 2$ are also true for $\mathscr{K}_{X_{0}}$ (with the appropriate interpretation). The coherence in particular implies that $H^{p}\left(\mathscr{K}_{X_{0}, x_{0}}\right)$ is a finite dimensional $\mathbb{C}$-vectorspace. For $\mathscr{K}_{X_{0}, x_{0}}^{*}=\Omega_{X_{0}, x_{0}}^{*}$ this is the well known theorem of Bloom and Herrera.

Lemma 3.2.1. Let $\left(\mathscr{K}^{*}, d\right)$ satisfy $\left(\mathrm{P}_{1}\right), \ldots,\left(\mathrm{P}_{4}\right)$. Assume that the canonical mapping of $\left(\mathrm{P}_{5}\right)$ is surjective and that $\mathscr{K}_{e r}\left(d^{1}: \mathscr{K}^{1} \rightarrow \mathscr{K}^{2}\right)$ is a torsion free $\mathcal{O}_{D}$-module. Then $\mathbb{R}^{1} f_{*}\left(\mathscr{K}^{*}\right)$ is a free $\mathscr{O}_{D}$-module.

Proof. Because of Theorem 2.1.1 it is sufficient to show that $H^{1}\left(\mathscr{K}_{x_{0}}^{*}\right)$ is a torsion free $\mathfrak{O}_{D, 0}$-module.

Let $\omega \in \mathscr{K}_{x_{0}}^{1}, d \omega=0$ and $f \omega=d g$ for some $g \in \mathscr{K}_{x_{0}}^{0}$. $g$ represents an element of $H^{0}\left(\mathscr{K}_{X_{0}, x_{0}}^{*}\right)$. By assumption there is a $g^{\prime} \in \mathscr{K}_{x_{0}}^{0}$ such that $d g^{\prime}=0$ and $g-g^{\prime}=f h$ for some $h \in \mathscr{K}_{x_{0}}^{0}$. Hence $f \omega=d g=f d h$ and therefore $f(\omega-d h)=0$. Now $\omega$ $-d h \in \mathscr{K} e r d^{1}$ since $\omega \in \mathscr{K} e r d^{1}$, hence $\omega=d h$ by assumption. The lemma is proved. 
We consider the local cohomology sheaves, (cf. [Gr]),

$$
\mathscr{H}_{x_{0}}^{0}\left(\mathscr{K}^{p}\right), p \geqq 0,
$$

which are coherent $\mathscr{O}_{X}$-subsheaves of $\mathscr{K}^{p}$. We shall freely make use of the fact that $\mathscr{H}_{x_{0}}^{0}\left(\mathscr{K}^{p}\right)$ coincides with the $\mathscr{O}_{D}$-torsion submodule of $\mathscr{K}^{p}$ since $\left.\mathscr{K}^{p}\right|_{X_{0}-\left\{x_{0}\right\}}$ is free after $\left(\mathrm{P}_{4}\right)$ of 2.1. $\mathscr{H}_{x_{0}}^{0}\left(\mathscr{K}^{*}\right)$ is a subcomplex of $\mathscr{K}^{\circ}$ and we can define the quotient complex

$$
\left(\overline{\mathscr{K}}^{\circ}, \bar{d}\right)=\left(\mathscr{K}^{*} / \mathscr{H}_{x_{0}}^{0}\left(\mathscr{K}^{*}\right), d / \mathscr{H}_{x_{0}}^{0}\left(\mathscr{K}^{*}\right)\right) .
$$

Lemma 3.2.2. If $\left(\mathscr{K}^{*}, d\right)$ satisfies $\left(\mathrm{P}_{1}\right), \ldots,\left(\mathrm{P}_{5}\right)$ the same holds for $\left(\overline{\mathscr{K}}^{\circ}, \bar{d}\right)$. Moreover, $\mathbb{R}^{1} f_{*}(\overline{\mathscr{K}})$ is a free $\mathscr{O}_{D}$-module which coincides on $D-\{0\}$ with $\mathbb{R}^{1} f_{*}\left(\mathscr{K}^{*}\right)$.

Proof. We have to check $\left(\mathrm{P}_{3}\right)$ and $\left(\mathrm{P}_{5}\right)$ for $\left(\overline{\mathscr{K}}^{*}, d\right)$, the other properties $\left(\mathrm{P}_{i}\right)$ being obvious.

$\left(\mathrm{P}_{3}\right):$ We show $\mathscr{H}^{0}\left(\overline{\mathscr{K}}^{\circ}\right)=\mathscr{H}^{0}\left(\mathscr{K}^{*}\right)=f^{-1} \mathscr{F}$.

Note that $\mathscr{H}_{x_{0}}^{0}\left(\mathscr{K}^{0}\right) \cap f^{-1} \mathscr{F}=0$, so we have an injection $f^{-1} \mathscr{H} \hookrightarrow \mathscr{H}^{0}\left(\overline{\mathscr{K}}^{\circ}\right) \subset \mathscr{K}^{0}$. Now let $g \in \mathscr{K}^{0}$ be such that $d g \in \mathscr{H}_{x_{0}}^{0}\left(\mathscr{K}^{1}\right)$. This implies $d g \mid X-\left\{x_{0}\right\}=0$, hence $g \mid X-\left\{x_{0}\right\} \in f^{-1} \mathscr{F}$. But $g \mid X-\left\{x_{0}\right\}$ has a unique prolongation to an element $g^{\prime} \in f^{-1} \mathscr{F}$. Since $g^{\prime}-g \in \mathscr{H}_{x_{0}}^{0}\left(\mathscr{K}^{0}\right)$ the claim is proved.

$\left(\mathrm{P}_{5}\right)$ : It suffices to show $\mathscr{H}^{0}\left(\overline{\mathscr{K}}_{\boldsymbol{X}_{0}}\right)=\mathscr{H}^{0}\left(\mathscr{K}_{\boldsymbol{X}_{0}}\right)$.

But this follows in the same manner as we proved $\left(\mathrm{P}_{3}\right)$ since $\mathscr{H}^{0}\left(\mathscr{K}_{X_{0}}\right)$ is a constant sheaf by $\left(\mathrm{P}_{5}\right)$. The rest of Lemma 3.2 .2 is true in view of Lemma 3.2.1.

For the following proposition we need some notations. Let $\mathscr{K}_{e r} d^{1}$ denote the kernel of $d^{1}: \mathscr{K}^{1} \rightarrow \mathscr{K}^{2}$. For an $\mathscr{O}_{D, t}$-module $M$ resp. an $\mathcal{O}_{D}$-sheaf $\mathscr{M}$ we set

$$
M(t)=M / \mathfrak{M}_{t} M \text { resp. } \mathscr{M}(t)=\mathscr{M}_{t} / \mathfrak{M}_{t} \mathscr{M}_{t}
$$

where $\mathfrak{M}_{t}$ denotes the maximal ideal of $\mathcal{O}_{D, t}$. For a sheaf $\mathscr{F}$ of $f^{-1} \mathcal{O}_{D}$-modules on $X$ we write

$$
h_{x_{0}, X_{0}}^{0}(\mathscr{F})=\operatorname{dim}_{\mathbb{C}}\left(H_{x_{0}}^{0}(\mathscr{F}) / f H_{x_{0}}^{0}(\mathscr{F})\right)
$$

where $H_{x_{0}}^{0}(-)$ denotes the stalk in $x_{0}$ of $\mathscr{H}_{x_{0}}^{0}(-)$.

Proposition 3.2.3. Assume that $\left(\mathscr{K}^{\circ}, d\right)$ satisfies $\left(\mathrm{P}_{1}\right), \ldots,\left(\mathrm{P}_{5}\right)$ and let $t \in D-\{0\}$. Then

$(*) \operatorname{dim}_{\mathbb{C}} \mathbb{R}^{1} f_{*}\left(\mathscr{K}^{*}\right)(0)-\operatorname{dim}_{\mathbb{C}} \mathbb{R}^{1} f_{*}\left(\mathscr{K}^{*}\right)(t)=\operatorname{dim}_{\mathbb{C}} H^{1}\left(H_{x_{0}}^{0}\left(\mathscr{K}^{*}\right)\right)(0)$.

In particular $\mathbb{R}^{1} f_{*}\left(\mathscr{K}^{*}\right)$ is free iff the complex $H_{x_{0}}^{0}\left(\mathscr{K}^{*}\right)$ is exact in degree 1. Moreover if $\mathscr{H}_{x_{0}}^{0}\left(\mathscr{K}^{0}\right)=0$ then the right hand side of $(*)$ is equal to $h_{x_{0}, x_{0}}^{0}\left(\mathscr{K}_{e r} d^{1}\right)$.

Proof. From the proof of $\left(\mathrm{P}_{3}\right)$ in Lemma 3.2.2 we deduce the following exact sequence

$$
0 \rightarrow H^{1}\left(H_{x_{0}}^{0}\left(\mathscr{K}^{*}\right)\right) \rightarrow H^{1}\left(\mathscr{K}_{x_{0}}^{*}\right) \rightarrow H^{1}\left(\overline{\mathscr{K}}_{x_{0}}^{*}\right)
$$


This is part of the cohomology sequence to $0 \rightarrow H_{x_{0}}^{0}\left(\mathscr{K}^{\circ}\right) \rightarrow \mathscr{K}_{x_{0}}^{*} \rightarrow \overline{\mathscr{K}}_{x_{0}}^{\circ} \rightarrow 0$. Since $H^{1}\left(\bar{K}_{x_{0}}^{*}\right)$ is free, $H^{1}\left(\mathscr{K}_{x_{0}}^{*}\right)$ maps onto a free module of the same rank. Hence

$$
\operatorname{dim}_{\mathbb{C}} H^{1}\left(\mathscr{K}_{x_{0}}^{\cdot}\right)(0)-\operatorname{dim}_{\mathbb{C}} H^{1}\left(\overline{\mathscr{K}}_{x_{0}}^{*}\right)(0)=\operatorname{dim}_{\mathbb{C}} H^{1}\left(H_{x_{0}}^{0}\left(\mathscr{K}^{*}\right)\right)(0) .
$$

The terms on the left are equal to $\operatorname{dim}_{\mathbb{C}} \mathbb{R}^{1} f_{*}\left(\mathscr{K}^{\circ}\right)(0)$ and $\operatorname{dim}_{\mathbb{C}} \mathbb{R}^{1} f_{*}\left(\overline{\mathscr{K}}^{*}\right)(0)$ respectively. By (3.2.2) we obtain

$$
\begin{aligned}
\operatorname{dim}_{\mathbb{C}} \mathbb{R}^{1} f_{*}\left(\overline{\mathscr{K}}^{*}\right)(0) & =\operatorname{dim}_{\mathbb{C}} \mathbb{R}^{1} f_{*}\left(\overline{\mathscr{K}}^{\circ}\right)(t) \\
& =\operatorname{dim}_{\mathbb{C}} \mathbb{R}^{1} f_{*}\left(\mathscr{K}^{*}\right)(t) .
\end{aligned}
$$

This proves the proposition.

Corollary 3.2.4. Assume that $\left(\mathscr{K}^{*}, d\right)$ consists of two terms only,

$$
0 \rightarrow \mathscr{K}^{0} \stackrel{d}{\longrightarrow} \mathscr{K}^{1} \rightarrow 0,
$$

and satisfies $\left(\mathrm{P}_{1}\right), \ldots,\left(\mathrm{P}_{5}\right)$. Assume moreover $\mathscr{H}^{0}\left(\mathscr{K}^{*}\right)=f^{-1} \mathcal{O}_{D}, \quad \mathscr{H}_{x_{0}}^{0}\left(\mathscr{K}^{0}\right)=0$ Then:

1) $\mathbb{R}^{1} f_{*}\left(\mathscr{K}^{*}\right) \cong f_{*} \mathscr{K}^{1} / d f_{*} \mathscr{K}^{0}$ is coherent,

2) $\mathbb{R}^{1} f_{*}\left(\mathscr{K}^{*}\right)_{0} \cong \mathscr{K}_{x_{0}}^{1} / d \mathscr{K}_{x_{0}}^{0}$,

3) $\operatorname{dim}_{\mathbb{C}} \mathscr{K}_{X_{0}, x_{0}}^{1} / d \mathscr{K}_{X_{0}, x_{0}}^{0}-\sum_{x \in S\left(X_{t}\right)} \operatorname{dim}_{\mathbb{C}} \mathscr{K}_{X_{t}, x}^{1} / d \mathscr{K}_{X_{t}, x}^{0}=\operatorname{dim}_{\mathbb{C}} H^{1}\left(X_{t}, \mathbb{C}\right)+h_{x_{0}, X_{0}}^{0}\left(\mathscr{K}^{1}\right)$

where $t \in D-\{0\}$.

(Note that $\left.\mathscr{K}_{X_{\mathrm{t}}}^{p}=\mathscr{K}^{p} /(f-t) \mathscr{K}^{p}\right)$.

Example 3.2.5. We can apply our theory also to the following, rather trivial situation:

Let $\varphi: \mathscr{G} \rightarrow \mathscr{F}$ be an injective, $\mathcal{O}_{X^{-}}$-linear (!) morphism of coherent $\mathscr{O}_{X^{-}}$ modules such that $\mathscr{F} / \varphi(\mathscr{G})$ is concentrated on $C(f)$. By the finite coherence theorem, $f_{*} \mathscr{F} / f_{*} \varphi(\mathscr{G})$ is coherent. We could have considered also the two terms complex $0 \rightarrow \mathscr{G} \rightarrow \mathscr{F} \rightarrow 0$, which satisfies $\left(\mathrm{P}_{1}\right), \ldots,\left(\mathrm{P}_{4}\right)$ (cf. remark after 2.1.1). Then Lemma 3.2.1 says: If $\mathscr{F}$ is $\mathscr{O}_{D}$-torsion free and if $\mathscr{G} \otimes_{\mathcal{O}_{X}} \mathcal{O}_{X_{0}} \rightarrow \mathscr{F} \otimes_{\mathscr{O}_{X}} \mathscr{O}_{X_{0}}$ is injective, then $f_{*} \mathscr{F} / f_{*} \varphi(\mathscr{G})$ is a free $\mathcal{O}_{D}$-module.

\section{Topology of Flat Families of Curves}

In this section we apply the machinery developped in Chap. 2 and 3 to show that $\mu$ is a measure for the vanishing (co-) homology. Moreover we study the topology of families of curves $f: X \rightarrow D$ with constant total Milnor number.

To do this we need the relative dualizing module of Grothendieck. Since we could not find an explicit and easy reference, we give a short summary of the definitions and results which we need in the sequel. For a general treatment see the work of Hartshorne $[\mathrm{H}]$ (and $\left[\mathrm{H}-\mathrm{K}_{1}\right]$ for the absolute case). 
For the study of $f: X \rightarrow D$ it is very useful to consider the family $\tilde{f}: \tilde{X} \rightarrow D$ where $n: \tilde{X} \rightarrow X$ is the normalization of $X$ and $\tilde{f}=f \circ n$. We recall the essential properties of $\tilde{f}$ which are due to Lejeune, Lê and Teissier.

\subsection{Relative Dualizing Sheaf and Normalization}

Relative Dualizing Sheaf. Let $f: X \rightarrow S$ be a Cohen-Macaulay morphism of complex spaces (i.e. $f$ is flat with Cohen-Macaulay fibers) of relative dimension $d$. We assume that $f$ is embeddable, i.e. there exists a commutative diagram

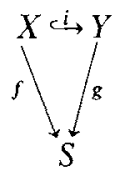

where $g$ is smooth of relative dimension $N$ and $i$ is an embedding of $X$ as a closed subspace of $Y$ of codimension $N-d$. (This is of course always possible locally on X.)

Then the relative dualizing sheaf $\omega_{x / s}$ exists and can be defined by

$$
\omega_{X / S}=i^{*} \mathscr{E}_{x} t_{\mathcal{O}_{X}}^{N-d}\left(i_{*} \mathscr{O}_{X}, \Omega_{Y / S}^{N}\right),
$$

where $\Omega_{Y / S}^{N}$ denotes the sheaf of relative holomorphic (Kähler) $N$-forms of $Y$ over $S$. Indeed $\omega_{X / S}$ is the unique non vanishing cohomology sheaf of the dualizing complex $f^{!} \mathscr{O}_{S}$ (cf. [H], p. 298, p. 192). If in particular $X$ itself is smooth over $S$ then $\omega_{X / S} \cong \Omega_{X / S}^{d}$. The definition of $\omega_{X / S}$ is independant of the embedding of $f$.

The most important property for our purposes is the local duality theorem for finite morphisms.

Theorem 4.1.1 ([H], Th. 6.7., p. 170). Let $f: X \rightarrow S, f^{\prime}: X^{\prime} \rightarrow S$ be two embeddable morphisms of complex spaces and $p: X^{\prime} \rightarrow X$ be a finite morphism with $f^{\prime}=f \circ p$. Assume that $\omega_{X / S}$ exists. Then $\omega_{X ; S}$ exists too and:

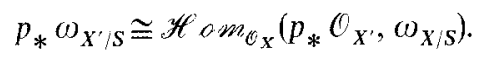

Remarks 4.1.2. 1) $\omega_{X / S}$ exists if $X \rightarrow S$ is Cohen-Macaulay. Since we will consider small representatives either of germs of reduced curves (over a point) or of germs of flat families of reduced curves over a disc, the existence of $\omega_{X / S}$ will be guaranteed.

2) Since $p$ is finite, $p_{*}$ is an exact functor and therefore knowledge of $p_{*} \omega_{X^{\prime} / S}$ is sufficient for the knowledge of $\omega_{X^{\prime} / S}$ : the $\mathcal{O}_{X^{\prime}}$-module structure of $\omega_{X^{\prime} / S}$ is defined by the natural action of $p_{*} \mathcal{O}_{X}$, on $\mathscr{H} a m_{\mathcal{O}_{X}}\left(p_{*} \mathcal{O}_{X^{\prime}}, \omega_{X / S}\right)$.

The properties of $\omega_{X / S}$ which we shall need are now easy consequences of Theorem 4.1.1 and of the definition of $\omega_{X / S}$.

Lemma 4.1.3. Let $f:(X, x) \rightarrow(D, 0)$ and $f^{\prime}:\left(X^{\prime}, x^{\prime}\right) \rightarrow(D, 0)$ be two germs of flat families of reduced curves, and $f: X \rightarrow D, f^{\prime}: X^{\prime} \rightarrow D$ sufficiently small representatives. 
Then:

(1) $\omega_{X / D} \otimes_{\mathcal{O}_{X}} \mathcal{O}_{X_{t}} \cong \omega_{X_{t}}$ for every $t \in D$, where $X_{t}=f^{-1}(t)$,

(2) $\omega_{X / D}$ is Cohen-Macaulay,

(3) Let $p: X^{\prime} \rightarrow X$ be a finite morphism satisfying $f \circ p=f^{\prime}$. There is a canonical morphism

$$
p_{*} \omega_{X^{\prime} / D} \rightarrow \omega_{X / D}
$$

which is injective if $p$ is generically injective.

Proof. (1), (2). Let $\pi:(X, x) \rightarrow(\mathbb{C} \times D, 0)$ be a finite, flat morphism where the second component is equal to $f$. By [EGA], IV, 0.16.4.8 it suffices to show that $\pi_{*} \omega_{X / D}$ is Cohen-Macaulay. But by 4.1 .1

$$
\pi_{*} \omega_{X / D} \cong \mathscr{H} \circ m_{\mathscr{C}_{\mathbb{C} \times D}}\left(\pi_{*} \mathcal{O}_{X}, \omega_{\mathbb{C} \times D / D}\right)
$$

Since $\omega_{\mathbb{C} \times D / D} \cong \Omega_{\mathbb{C} \times D / D}^{1} \cong \mathcal{O}_{\mathbb{C} \times D}$, the right hand side is isomorphic to $\mathscr{H}_{0} m_{\mathbb{C} \times D}\left(\pi_{*} \mathcal{O}_{X}, \mathcal{O}_{\mathbb{C} \times D}\right)$. Hence $\pi_{*} \omega_{X / D}$ is Cohen-Macaulay because $\pi_{*} \mathcal{O}_{X}$ is a locally-free $\mathcal{O}_{\mathbb{C} \times D^{\prime}}$-module. Again by 4.1.1 we obtain $\pi_{*} \omega_{X / D} \otimes_{\mathbb{O} \mathbb{C} \times D} \mathcal{O}_{\mathbb{C} \times\{t\}} \cong q_{*} \omega_{X_{t}}$, where $q=\left.\pi\right|_{X_{t}}: X_{t} \rightarrow \mathbb{C} \times\{t\}$. This proves (1).

(3) The structural morphism $\mathscr{O}_{X} \rightarrow p_{*} \mathcal{O}_{X}$ induces a morphism

$$
p_{*} \omega_{X^{\prime} / D} \cong \mathscr{H} \circ m_{C_{X}}\left(p_{*} \mathscr{O}_{X}, \omega_{X / D}\right) \rightarrow \mathscr{H} \sigma_{0 m_{D_{X}}}\left(\mathcal{O}_{X}, \omega_{X / D}\right) \cong \omega_{X / D}
$$

If $p$ is generically $1-1$ then $p_{*} \mathcal{O}_{X^{\prime}} / \mathcal{O}_{X}$ is concentrated on a lower dimensional analytic subset of $X$. Hence

$$
\mathscr{K} \operatorname{er}\left(p_{*} \omega_{X^{\prime} / D} \rightarrow \omega_{X / D}\right)=\mathscr{H}_{\operatorname{com}_{O_{X}}}\left(p_{*} \mathcal{O}_{X^{\prime}} / \mathcal{O}_{X}, \omega_{X / D}\right)=0
$$

since $\omega_{X / D}$ is Cohen-Macaulay.

Normalization. Let $f:\left(X, x_{0}\right) \rightarrow(D, 0)$ be the germ of a flat family of reduced curves. Let

$$
n:\left(\tilde{X}, \tilde{x}_{0}\right) \rightarrow\left(X, x_{0}\right)
$$

denote the normalization of the surface $\left(X, x_{0}\right)$; here $\tilde{x}_{0}=n^{-1}\left(x_{0}\right)$ consists of a finite number of points (as many as there are irreducible components of $\left(X, x_{0}\right)$ ) so that $\left(\tilde{X}, \tilde{x}_{0}\right)$ is a multi-germ of normal surfaces.

The essential properties of the composition

$$
\tilde{f}=f \circ n:\left(\tilde{X}, \tilde{x}_{0}\right) \rightarrow(D, 0)
$$

are given in the following theorem (due as stated to $\mathrm{B}$. Teissier [ $\mathrm{Te}_{3}$ ] 1.3.2, maturing the earlier result in [L-L-T]). To state it we need the following notation: Let $\tilde{f}: \tilde{X} \rightarrow D$ (resp. $f: X \rightarrow D$ ) be sufficiently small representatives of the corresponding map germs $f$ (resp. $f$ ) and put $X_{t}=f^{-1}(t), \tilde{X}_{t}=f^{-1}(t), t \in D$. $\bar{X}_{t}$ denotes the normalization of $X_{t}$. 
Theorem 4.1.4 (Lejeune, Lê,Teissier). (1) $\left.\tilde{f}:\left(\tilde{X}, \tilde{x}_{0}\right) \rightarrow(D, 0)\right)$ is flat.

(2) For sufficiently small $t \in D-\{0\}$ we have $\tilde{X}_{t}=\bar{X}_{t}$ and

$$
\delta\left(X_{0}\right)-\delta\left(X_{\imath}\right)=\delta\left(\tilde{X}_{0}\right)
$$

where $\delta(C)=\sum_{x \in S(C)} \delta(C, x)$ and $S(C)$ denotes the singular set of $C$ for any curve $C$.

Proof. $\left(\tilde{X}, \tilde{x}_{0}\right)$ is a normal surface, hence Cohen-Macaulay. Since the fibers of $\tilde{f}$ are 1-codimensional, $\tilde{f}$ is a nonzero divisor and therefore flat. As a normal surface, $\left(\tilde{X}, \tilde{x}_{0}\right)$ has only finitely many singularities. By Bertini's or Sard's theorem, $\tilde{f}$ is singular at most in $\tilde{x}_{0}$ for sufficiently small $D$. So $\tilde{X}_{t}$ is smooth if $t \in D-\{0\}$, and by the universal property of normalization, $\tilde{X}_{t}=\bar{X}_{t}$.

By Remark 3.2.5 $\tilde{f}_{*} \mathcal{O}_{\tilde{X}} / f_{*} \mathcal{O}_{X}$ is free on $D$. This implies the dimension formula, observing that $\left(\tilde{X}_{0}, \tilde{x}_{0}\right)$ and $\left(X_{0}, x_{0}\right)$ have the same normalization $\left(\bar{X}_{0}, \bar{x}_{0}\right)$.

Definition 4.1.5. (cf. $\left[\mathrm{Te}_{3}\right]$ ). (1) $f: X \rightarrow D$ admits a normalization in family if $\tilde{X}_{t}$ is smooth for all $t \in D$.

(2) Assume that $f:\left(X, x_{0}\right) \rightarrow(D, 0)$ admits a section $\sigma:(D, 0) \rightarrow\left(X, x_{0}\right)$ such that $X_{t}-\sigma(t)$ is smooth for all $t \in D$. Then $f$ admits a simultaneous (resp. weak simultaneous) resolution if $f$ admits a normalization in family and

$$
\begin{array}{rlrl}
n^{-1}(\sigma(D)) \cong D \times n^{-1}(\sigma(0)) & & \text { (over } D) \\
\text { (resp. } n^{-1}(\sigma(D))_{\text {red }} & \cong D \times n^{-1}(\sigma(0))_{\text {red }} & & \text { (over } D),
\end{array}
$$

where the index "red" means the associated reduced space,

Remark 4.1.6. (cf. $\left[\mathrm{Te}_{3}\right]$ ). (1) Normalization in family is equivalent to $\delta\left(X_{0}\right)$ $=\delta\left(X_{t}\right)$ by Theorem 4.1.4. In this case we say also that $f:\left(X, x_{0}\right) \rightarrow(D, 0)$ is a $\delta$ constant family.

(2) Weak simultaneous resolution is equivalent to: $f$ admits a normalization in family and the number of branches of $\left(X_{t}, \sigma(t)\right)$ is constant for all $t \in D$. If in addition the multiplicity $m\left(X_{t}, \sigma(t)\right)$ is constant, this is equivalent to simultaneous resolution.

\subsection{Milnor Number and Vanishing Cohomology}

Before we can prove the main result of this section we have to explain how to extend the mapping $d: \mathcal{O}_{X_{0}, x_{0}} \stackrel{d}{\longrightarrow} \Omega_{X_{0}, x_{0}}^{1} \rightarrow \omega_{X_{0}, x_{0}}$ constructed in 1.1 to the relative case.

Proposition 4.2.1. Let $f:\left(X, x_{0}\right) \rightarrow(D, 0)$ be a flat family of reduced curves and $f: X \rightarrow D$ a small representative. Then there exists a natural morphism

$$
c_{X / D}: \Omega_{X / D}^{1} \rightarrow \omega_{X / D}
$$

such that the analytic restriction $c_{X_{t}, x}=c_{X / D} \otimes_{\mathcal{O}_{X}} \mathcal{O}_{X_{t}, x}: \Omega_{X_{t}, x}^{1} \rightarrow \omega_{X_{t}, x}$ is the morphism $\Omega_{X_{t}, x}^{1} \rightarrow \omega_{X_{t}, x}$ constructed in $1.1\left(t \in D, x \in X_{t}\right)$. 
Proof. Let $n: \tilde{X} \rightarrow X$ be the normalization of $X, \tilde{x}_{0}=n^{-1}\left(x_{0}\right)$. The critical set $C(\tilde{f})$ of $\tilde{f}=f \circ n$ has codimension at least two in $\tilde{X}$ (and by taking small enough representatives we may assume $\left.C(\tilde{f}) \subset \tilde{x}_{0}\right)$. Let $i: \tilde{X}-C(\tilde{f}) \hookrightarrow \tilde{X}$ denote the inclusion. We define $c_{X / D}$ to be the composition

$$
c_{X / D}: \Omega_{X / D}^{1} \stackrel{\alpha \tilde{X} / X}{\longrightarrow} n_{*} \Omega_{\tilde{X} / D}^{1} \rightarrow n_{*} i_{*} i^{*} \Omega_{\tilde{X} / D}^{1} \cong n_{*} i_{*} i^{*} \omega_{\tilde{X} / D}=n_{*} \omega_{\tilde{X} / D} \stackrel{\beta \tilde{X} / X}{\longrightarrow} \omega_{X / D} .
$$

All the mappings in the diagram are canonically defined. We have $i^{*} \Omega_{\bar{X} / D}^{1}$ $\cong i^{*} \omega_{\tilde{X} / D}$ since $\tilde{f} \circ i$ is smooth and $\omega_{\tilde{X} / D}=\mathrm{i}_{*} \mathrm{i}^{*} \omega_{\tilde{X} / D}$. This follows from the exact sequence of local cohomology since $\omega_{\tilde{X} / D}$ is Cohen-Macaulay by lemma 4.1.3 (2) (cf. $[\mathrm{Ba}]$ ).

$c_{X / D}$ is natural in the sense that if $p: X^{\prime} \rightarrow X$ is a finite morphism with $f \circ p$ $=f^{\prime}$, then

$$
c_{X / D}=\beta_{X^{\prime} / X} \circ p_{*} c_{X^{\prime} / D^{\prime}} \circ \alpha_{X^{\prime} / X} .
$$

Using this fact it is now a matter of chasing the obvious diagrams to show that the analytic restriction of $c_{X / D}$ on each fiber coincides with the morphism constructed in 1.1.

Remark. The definition of $c_{X / D}$ is possible in a much more general context (cf. $[\mathrm{El}])$.

Now we assume that the representative $f: X \rightarrow D$ of $f:\left(X, x_{0}\right) \rightarrow(D, 0)$ is "good", i.e. satisfies the conditions (a)-(c) of 2.1. Then $H^{0}\left(X_{t}, \mathbb{C}\right)=\mathbb{C}$ and $\operatorname{dim}_{\mathbb{C}} H^{1}\left(X_{t}, \mathbb{C}\right), t \in D-\{0\}$, is independent of the chosen representative. Since $X_{0}$ is contractible we call $H^{1}\left(X_{t}, \mathbb{C}\right), t \in D-\{0\}$, the vanishing cohomology of the family $f:\left(X, x_{0}\right) \rightarrow(D, 0)$. The following theorem shows that $\mu$ measures exactly the vanishing cohomology. For the proof of that theorem we need nearly everything we have proved so far.

We use the following notations $(t \in D)$ :

$$
\begin{aligned}
& \mu_{t}=\mu\left(X_{t}\right)=\sum_{x \in X_{t}} \mu\left(X_{t}, x\right), \\
& \delta_{t}=\delta\left(X_{t}\right)=\sum_{x \in X_{t}} \delta\left(X_{t}, x\right),
\end{aligned}
$$

where we have to sum of course only over the finitely many singular points of $X_{t}$.

Theorem 4.2.2. Let $f: X \rightarrow D$ be a good representative of a flat family $f:\left(X, x_{0}\right) \rightarrow(D, 0)$ of reduced curves. Then for all $t \in D:$

(1) $X_{t}$ is connected,

(2) $\mu_{0}-\mu_{t}=\operatorname{dim}_{\mathbb{C}} H^{1}\left(X_{t}, \mathbb{C}\right)$,

(3) $\mu_{0}-\mu_{t} \geqq \delta_{0}-\delta_{t}$.

Corollary 4.2.3 (1) (Bassein [Ba]). If $X_{t}$ is smooth for $t \neq 0$ then

$\mu\left(X_{0}, x_{0}\right)=\operatorname{dim}_{\mathbb{C}} H^{1}\left(X_{t}, \mathbb{C}\right)$.

(2) The function $s \rightarrow \mu_{s}, s \in S_{\text {red }}$ is upper semi-continuous on the reduced base $S_{\mathrm{red}}$ of the semi-universal deformation of $\left(X_{0}, x_{0}\right)$. 
Proof (of the Theorem). (1), (2). We define $\omega_{X / D}$ to be the complex with two non vanishing terms,

$$
\omega_{X / D}^{\cdot}: 0 \rightarrow \mathcal{O}_{X} \stackrel{c_{X / D^{\circ d}}}{\longrightarrow} \omega_{X / D} \rightarrow 0
$$

where $d: \mathscr{O}_{X} \rightarrow \Omega_{X / D}^{1}$ denotes the universal derivation. Then $\mathscr{H}^{0}\left(\omega_{X / D}^{*}\right)=f^{-1} \mathcal{O}_{D}$ and $\mathscr{H}^{0}\left(\omega_{X / D}^{*} / f \omega_{X / D}\right)=\mathbb{C}$, so it follows immediately that $\omega_{X / D}^{\cdot}$ satisfies the properties $\left(\mathrm{P}_{1}\right), \ldots,\left(\mathrm{P}_{4}\right)$ of 2.1 and $\left(\mathrm{P}_{5}\right)$ of 3.2. Since $\omega_{X / D}$ is Cohen-Macaulay (Lemma 4.1.3) it is torsion free. Hence by Lemma 3.2.1, $\mathbb{R}^{1} f_{*} \omega_{X / D}$ is a free ${ }^{O_{D}}{ }^{-}$ module. Therefore the Gysin sequence 3.2.3 (3) and Proposition 4.2.1 imply

$$
\begin{aligned}
\operatorname{dim}_{\mathbb{C}} H^{j}\left(X_{t}, \mathbb{C}\right) & =\operatorname{dim}_{\mathbb{C}} \omega_{X_{0}, x_{0}} / d \mathcal{O}_{X_{0}, x_{0}}-\sum_{x \in S\left(X_{t}\right)} \operatorname{dim}_{\mathbb{C}} \omega_{X_{\mathfrak{t}}, x} / d \mathcal{O}_{X_{t}, x} \\
& =\mu_{0}-\mu_{t} .
\end{aligned}
$$

To see that $X_{t}$ is connected, we consider $\mathbb{R}^{0} f_{*}\left(\omega_{X / D}^{\circ}\right)_{t} \cong H^{0}\left(X_{t}, \underset{\mathbb{C}}{\mathbb{C}} \otimes \mathcal{O}_{D, t}\right.$ (3.1.1). Since $\mathbb{R}^{0} f_{*}\left(\omega_{X / D}^{\circ}\right)$ is coherent with a free stalk of rank 1 over 0 , the other stalks are also free of rank 1 . Hence $H^{0}\left(X_{t}, \mathbb{C}\right)=\mathbb{C}$ and $X_{t}$ is connected.

(3) Now we consider the family $\tilde{f}:\left(\tilde{X}, \tilde{x}_{0}\right) \rightarrow(D, 0)$, where $\tilde{x}_{0}=n^{-1}\left(x_{0}\right)$ $=\left\{\tilde{x}_{1}, \ldots, \tilde{x}_{\rho}\right\}$. Since we can consider each germ $\tilde{f}:\left(\tilde{X}, \tilde{x}_{i}\right) \rightarrow(D, 0) \quad(i=1, \ldots, \rho)$ separately, we deduce as in (1)

$$
\mu\left(\tilde{X}_{0}\right)-\mu\left(\tilde{X}_{t}\right)=\operatorname{dim}_{\mathbb{C}} H^{1}\left(\tilde{X}_{t}, \mathbb{C}\right) .
$$

By Theorem 4.1.4 (2), $\tilde{X}_{t}=\bar{X}_{t}$ is smooth, hence $\mu\left(\tilde{X}_{t}\right)=0$ if $t \neq 0$. Moreover we have proved the following:

$$
\begin{gathered}
\mu\left(\tilde{X}_{0}\right)=\sum_{i=1}^{\rho}\left(2 \delta\left(\tilde{X}_{0}, \tilde{x}_{i}\right)-r\left(\tilde{X}_{0}, \tilde{x}_{i}\right)+1\right), \\
\delta\left(\tilde{X}_{0}, \tilde{x}_{i}\right) \geqq r\left(\tilde{X}_{0}, \tilde{x}_{i}\right)-1, \\
\delta\left(\tilde{X}_{0}\right)=\delta\left(X_{0}\right)-\delta\left(X_{i}\right) .
\end{gathered}
$$

Since for any curve $C, \operatorname{dim}_{\mathbb{C}} H^{1}(C, \mathbb{C}) \geqq \operatorname{dim}_{\mathbb{C}} H^{1}(\bar{C}, \mathbb{C})$, we obtain:

$$
\mu_{0}-\mu_{t}=\operatorname{dim}_{\mathbb{C}} H^{1}\left(X_{t}, \mathbb{C}\right) \geqq \operatorname{dim}_{\mathbb{C}} H^{1}\left(\tilde{X}_{t}, \mathbb{C}\right)=\mu\left(\tilde{X}_{0}\right) \geqq \delta\left(\tilde{X}_{0}\right)=\delta_{0}-\delta_{t} .
$$

The next result, which is a corollary of Theorem 4.2 .2 points already in the direction of equisingularity.

Theorem 4.2.4. Let $f: X \rightarrow D$ be as in Theorem 4.2.2. Then the following conditions are equivalent:

(1) $\mu_{t}$ is constant for all $t \in D$,

(2) $\delta_{t}$ and $r_{t}^{\prime}=\sum_{x \in X_{t}}\left(r\left(X_{t}, x\right)-1\right)$ are constant for all $t \in D$,

(3) $H^{1}\left(X_{v}, \mathbb{C}\right)=0$ for all $t \in D$,

(4) $X_{t}$ is contractible for all $t \in D$. 
Proof. The equivalences $(1) \Leftrightarrow(2) \Leftrightarrow(3)$ follow immediately from Theorem 4.2.2 and from $\mu_{t}=2 \delta_{t}+r_{t}^{\prime}$. The only thing to show is (3) $\Rightarrow(4)$.

But $H^{1}\left(X_{t}, \mathbb{C}\right)=0$ implies $H^{1}\left(\bar{X}_{t}, \mathbb{C}\right)=0$, so that $\bar{X}_{t}$ is the disjoint union of dises by the classification of open Riemann surfaces. Therefore $X_{t}$ is homeomorphic to a union of discs, each corresponding to a (global) irreducible component of $X_{t}$, with certain points identified. Now consider the corresponding graph of this configuration. Since its number of cycles is equal to $\operatorname{dim}_{\mathbb{C}} H^{1}\left(X_{t}, \mathbb{C}\right)$ $-\operatorname{dim}_{\mathbb{C}} H^{1}\left(\bar{X}_{t}, \mathbb{C}\right)$ by Mayer-Vietoris, there are no cycles and hence $X_{t}$ is contractible.

\section{Topological Equisingularity}

We shall apply the results of the preceeding section to show that " $\mu=$ constant" is a necessary and sufficient condition for a family of curves to be topological equisingular in a certain sense. Since we consider curves which are embedded with arbitrarily high codimension we have to explain what we mean by "equisingular". This is discussed in 5.1. Moreover, since in a $\mu$-constant family of non plane curves the singularity may split (cf. Example 7.2.5), we consider only families of curves which have at most one singularity. The situation for plane curves is reviewed in 5.3.

\subsection{Equisingularity in Higher Codimension}

Let $f:\left(X, x_{0}\right) \rightarrow(D, 0)$ be a flat family of reduced curves and $f: X \rightarrow D$ a good representative in the sense of 2.1. In particular $X \subset B_{0} \times D$, where $B_{0} \subset \mathbb{C}^{n}$ is a small ball with center $x_{0}$, and $f$ is the restriction of the projection on the disc $D \subset \mathbb{C}$. We assume that there is a section $\sigma: D \rightarrow X, \sigma(0)=x_{0}$, of $f$ such that $X_{t}-\sigma(t)=\left(X \cap B_{0} \times\{t\}\right)-\sigma(t)$ is smooth. Sometimes we shall identify $B_{0}$ and $B_{0} \times\{t\}$ if there is no risk of confusion.

If $n=2$, the topological type of $\left(X_{0}, x_{0}\right)$ is defined to be the homeomorphic type of the pair $\left(B_{0}, X_{0}\right)$ for sufficiently small $B_{0}$. It is also characterized by the topological type of the link $\left(\partial B_{0}, \partial B_{0} \cap X_{0}\right)$ and this in turn is equivalent to the numerical data consisting of the Puiseux pairs of each branch of $\left(X_{0}, x_{0}\right)$ and of all intersection multiplicities of pairwise distinct branches. Moreover, given two germs of topological equisingular plane curves (i.e. having the same topological type), they can be embedded into a 1-parameter family as above. There exist many other characterizations of the topological type, mostly due to Zariski, who initiated the whole study of equisingularity (cf. $\left[\mathrm{Z}_{1}\right]$ ).

A simple numerical criterion for topological equisingularity (still in the case $n=2)$ was given by Lê and Ramanujam [L-R]: If $\mu\left(X_{t}, \sigma(t)\right)$ is constant, then the topological type of $\left(X_{t}, \sigma(t)\right)$ is constant. (This is true for all isolated hypersurface singularities of dimension $m \neq 2$; the case $m=2$ is unknown). The result of LêRamanujam was complemented by Timourian [Ti], who showed that " $\mu$ $=$ constant" implies even topological triviality. Note that this criterion refers to a family; the Milnor number alone does not characterize the topological type. It 
should be noted that " $\mu=$ constant" is also a necessary condition for constant topological type and topological triviality.

All this seems to be out of order if we consider curves in $\mathbb{C}^{n}, n \geqq 3$. It is well known that in this case the link, which consists of a certain number of disjoint circles in a sphere of dimension $2 n-1$, is trivial. Therefore the only topological invariant of a germ of a curve is the number of these circles, i.e. the number of irreducible components of the germ. In other words: Let $\left(X_{0}, x_{0}\right)$, $\left(X_{0}^{\prime}, x_{0}\right) \subset\left(\mathbb{C}^{n}, x_{0}\right)(n \geqq 3)$ be two germs of curves. Then there exist small balls $B_{0}$, $B_{0}^{\prime} \subset \mathbb{C}^{n}$ with center $x_{0}$ such that $\left(B_{0}, X_{0}\right)$ and $\left(B_{0}^{\prime}, X_{0}^{\prime}\right)$ are homeomorphic, iff $\left(X_{0}, x_{0}\right)$ and $\left(X_{0}^{\prime}, x_{0}\right)$ have the same number of branches.

But the situation is different if we consider a family of curves $f: X \rightarrow D$ as above. First of all, it makes sense to ask for a numerical criterion for the family to be topologically trivial. On the other hand we may also ask for conditions, such that for a sufficiently small fixed ball $B_{0}$ there exist homeomorphisms between $\left(B_{0}, X_{0}\right)$ and $\left(B_{0}, X_{t}\right)$ for all $t \in D$. Note, that constant topological type requires only that for each $t \in D$ there exists a ball $B_{t}$ with center $\sigma(t)$ and a homeomorphism between $\left(B_{0}, X_{0}\right)$ and $\left(B_{t}, X_{t}\right)$. But the size of $B_{t}$ may tend to 0 if $t$ tends to 0 . For plane curve this makes no difference: Constant topological type is equivalent to the existence of homeomorphisms between $\left(B_{0}, X_{0}\right)$ and $\left(B_{0}, X_{t}\right)$ for all $t$ by Theorem 5.2.2 and what we said above.

Another (related) fundamental difference from the case of plane curves is the existence of a "vanishing fold" in a $\mu$-constant family. This means the following. Let $B_{t} \subset \mathbb{C}^{n}$ be the biggest possible ball with center $\sigma(t)$ such that each sphere contained in $B_{t}$ with center $\sigma(t)$ intersects $X_{t}$ transversally. Let $\varepsilon_{t}\left(0<\varepsilon_{t} \leqq \infty\right)$ be the radius of $B_{t}$. We say that the family $\left(X_{t}\right)_{t \in D}$ has a vanishing fold if $\lim _{t \rightarrow 0} \varepsilon_{t}=0$ (cf. Fig. 1).

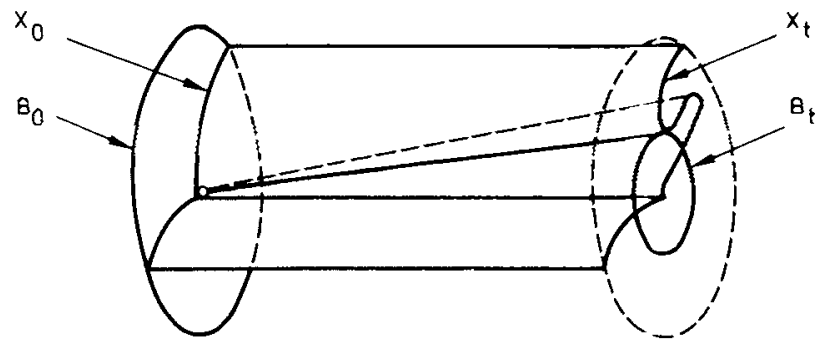

Fig. 1

Since $\mu$-constant families of plane curves satisfy the Whitney conditions along $\sigma(D)$, such vanishing folds cannot occur. But for arbitrary curves this may happen. This is related to the fact that the multiplicity need not be constant. See Example 7.2.1 which has a vanishing fold and therefore does not satisfy Whitney conditions (cf. also 6.2.7).

These remarks show that in higher codimension the Milnor number is a weaker invariant than in codimension 1 . The reason is that the topology of the embedded curves contains less informaiton and that $\mu$ reflects exactly the topological behaviour of a flat family of curves (Theorem 5.2.2). 


\subsection{Constant Milnor Number is Equivalent to Topological Triviality}

We keep the notations and assumptions of the beginning of 5.1. As always $\mu$ denotes the Milnor number, $r$ the number of branches and $\delta$ the $\delta$-invariant (cf. 2.1).

Lemma 5.2.1. Let $f: X \rightarrow D$ be a good representative of a flat family of reduced curves. Let $\sigma: D \rightarrow X$ be a section of $f$ such that $\mu\left(X_{t}, \sigma(t)\right)$ is constant. Then $X_{t}-\sigma(t)$ is smooth.

Proof. This follows immediately from Theorem 4.2.2 and Proposition 1.2.2.

Theorem 5.2.2. Let $f: X \rightarrow D$ be a good representative of a flat family of reduced curves with section $\sigma: D \rightarrow X$ such that $X_{t}-\sigma(t)$ is smooth for each $t \in D$. The following conditions are equivalent.

(1) $\mu\left(X_{1}, \sigma(t)\right)$ is constant for $t \in D$,

(2) $\delta\left(X_{t}, \sigma(t)\right)$ and $r\left(X_{t}, \sigma(t)\right)$ are constant for $t \in D$,

(3) $\operatorname{dim}_{\mathbb{C}} H^{1}\left(X_{t}, \mathbb{C}\right)=0$ for $t \in D$,

(4) $f: X \rightarrow D$ admits a weak simultaneous resolution (cf. 4.1.5(2)),

(5) There exists a homeomorphism between $\left(B_{0}, X_{0}\right)$ and $\left(B_{0}, X_{t}\right)$ for each $t \in D$,

(6) $f: X \rightarrow D$ is topologically trivial, i.e. there is a homeomorphism $h$ : $X \stackrel{\approx}{\rightarrow} X_{0} \times D$ such that $f=\pi \circ h$ where $\pi: X_{0} \times D \rightarrow D$ is the projection.

Proof. Consider the following implications:

$$
\begin{gathered}
(1) \Leftrightarrow(2) \Leftrightarrow(3) \Leftrightarrow(5) \\
\Downarrow \Uparrow \\
(4) \Rightarrow(6) .
\end{gathered}
$$

By Theorem 4.2.2 and by Remark 4.1.6 we need only show (4) $\Rightarrow(6)$ and $(3) \Rightarrow(5)$. (4) $\Rightarrow(6)$ : Let $n: \tilde{X} \rightarrow X$ and $\bar{X}_{0} \rightarrow X_{0}$ denote the respective normalizations. The hypothesis implies that $\tilde{f}=f \circ n: \tilde{X} \rightarrow D$ is smooth. Since the restriction of $\tilde{f}$ to the boundary of $\tilde{X}$ has maximal rank (by $2.1 \mathrm{c}$ ) it follows from the fibration theorem of Ehresman that there is a commutative diagram

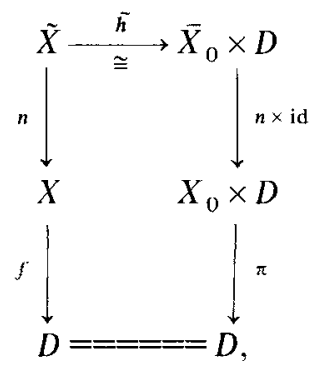

where $\tilde{h}$ is a diffeomorphism.

We have in particular that the irreducible components $X^{i}$ of $X$ are in one to one correspondance with the irreducible components $X_{0}^{i}$ of $X_{0}$. Since $r\left(X_{t}, \sigma(t)\right)$ is constant we deduce that each $X^{i}$ is locally irreducible along $\sigma(D)$. Therefore $n$ (resp. $n \times$ id) maps each connected component of $\tilde{X}$ (resp. of $\bar{X}_{0} \times D$ ) homeomorphically to an irreducible component of $X$ (resp. of $\left.X_{0} \times D\right)$. In this way 
we obtain for each $i$ a homeomorphism

$$
h^{i}=\left.(n \times \mathrm{id}) \circ \tilde{h} \cdot n^{-1}\right|_{X^{1}}: X^{i} \stackrel{\approx}{\longrightarrow} X_{0}^{i} \times D
$$

which respects the fibers. But the $h^{i}$ coincide on the common intersection $\sigma(D)$ of the $X^{i}$. Therefore (6) follows.

(3) $\Rightarrow(5)$. The case $\left(X_{0}, x_{0}\right) \subset\left(\mathbb{C}^{n}, x_{0}\right)$ with $n=2$ is essential due to Lê and Ramanujam [L-R]: consider $f: X \hookrightarrow B_{0} \times D \stackrel{\pi}{\longrightarrow} D$ and let $F(x, t)$ be the equation of $X$ in $B_{0} \times D$. We consider $F$ as a mapping from $B_{0} \times D \rightarrow \mathbb{C}$ and put $N_{t}$ $=F_{t}^{-1}(S) \subset B_{0}$ where $S \subset \mathbb{C}$ is a small disc and $F_{t}(x)=F(x, t)$. Then the pair $\left(N_{0}, x_{0}\right)$ is homeomorphic to the cone over $\left(\partial N_{0}, \partial X_{0}\right)$ such that $x_{0}$ is the vertex of the cone. Here $\partial N_{0}=F^{-1}(\partial \bar{S}) \cup\left(F^{-1}(\bar{S}) \cap \partial \bar{B}_{0}\right)$ and $\partial X_{0}=\bar{X}_{0} \cap \partial B_{0}$. This is due to Milnor [Mi]. Lê-Ramanujan show that the same is true for $\left(N_{t}, X_{t}\right)$ and that $\left(\partial N_{0}, \partial X_{0}\right)$ and $\left(\partial N_{t}, \partial X_{t}\right)$ are homeomorphic. By the conic structure this homeomorphism can be extended to a homeomorphism between $\left(N_{0}, X_{0}\right)$ and $\left(N_{t}, X_{t}\right)$. The conic structure of $\left(N_{0}, X_{0}\right)$ (resp. $\left(N_{t}, X_{t}\right)$ ) is defined by integrating a certain vector field, which can be extended all over $B_{0}$. Integrating both extended vector fields we can extend the homeomorphism $\left(N_{0}, X_{0}\right) \rightarrow\left(N_{t}, X_{t}\right)$ to a homeomorphism $\left(B_{0}, X_{0}\right) \rightarrow\left(B_{0}, X_{t}\right)$. (For similar, detailed arguments cf. [Ti].)

Now assume $n \geqq 3$ : Since $X_{t}$ is transversal to $\partial B_{0}$ for all $t \in D$, it follows from the fibration theorem of Ehresman that the restriction of $f$ to $\partial X$ is differentially trivial. This implies that $\partial X_{0}$ and $\partial X_{t}$ are isotopically embedded in $\partial B_{0}$. But since the real codimension of $\partial X_{t}$ in $\partial B_{0}$ is greater than or equal to 4 , this isotopy can be extended to an ambient isotopy of $\partial B_{0}$ (cf. [Hu], Th. 10.1). By assumption, $\operatorname{dim}_{\mathbb{C}} H^{1}\left(X_{t}, \mathbb{C}\right)=0$, so that $X_{t}$ is contractible and homeomorphic to a one point union of discs since $X_{t}-\sigma(t)$ is smooth (cf. the proof of Theorem 4.2.4). The same holds for $X_{0}$ and therefore $X_{0}$ and $X$, are homeomorphic to the cones over $\partial X_{0}$ and $\partial X_{1}$ respectively. The theorem of Lickorish [Li] about the unknotting of cones implies that the homeomorphism $\left(\partial B_{0}, \partial X_{0}\right) \rightarrow\left(\partial B_{0}, \partial X_{t}\right)$ can be extended to a homeomorphism of $B_{0}$ which sends $X_{0}$ to $X_{t}$.

\subsection{Comparison to the Case of Plane Curves}

As mentioned in 5.1, the concept of equisingularity was introduced about 1965 by $\mathrm{O}$. Zariski. Today there is a well-understood theory of equisingularity for plane curves, starting with the results of $\mathrm{O}$. Zariski $\left[Z_{1}\right]$ further developed among others - by $\mathrm{O}$. Zariski himself, H. Hironaka, M. Lejeune, Lê Dũng Tràng and B. Teissier. A short summary of equisingularity for plane curves can be found in $\left[\mathrm{Te}_{2}\right]$. Also good discussions of the results and the problems of equisingularity for higher dimensional families in codimension one are available (cf. $\left[Z_{2}, Z_{3}, \mathrm{Te}_{1}, \mathrm{~L}-\mathrm{T}\right]$ ).

For the readers convenience we give here a short summary of known results for plane curves to outline the historical traces leading to 5.2.2 and to exhibit other possible notions of equisingularity than the topological one given above.

The main results can be summarized as follows. 
Theorem 5.3.1 (cf. $\left[\mathrm{Te}_{3}\right],\left[\mathrm{Te}_{2}\right]$ 3.7, [L-R]). Let $f: X \rightarrow D$ be as in 5.2.2 and assume furthermore that $X_{0}$ is a plane curve. Then $X_{t}$ is also plane curve for all $t \in D$ and the following conditions are equivalent:

(1) ... (6) as in 5.2.2,

(7) $\mu\left(X_{t}, \sigma(t)\right)$ and $m\left(X_{t}, \sigma(t)\right)$ (the multiplicity of $X_{t}$ in $\sigma(t)$ ) are constant for $t \in D$,

(8) the pair $(X-\sigma(D), \sigma(D))$ satisfies the conditions a) and b) of Whitney (cf. $\left[\mathrm{Te}_{3}\right]$ ),

(9) $f: X \rightarrow D$ is Lipschitzian equisaturated at every point of $\sigma(D)\left(\mathrm{cf} .\left[\mathrm{Te}_{3}\right]\right.$ ),

(10) For every $t \in D$ there exists a bijection between the branches of $\left(X_{t}, \sigma(t)\right)$ and $\left(X_{0}, \sigma(0)\right)$ resp. such that the Puiseux pairs of corresponding branches and their multiplicities of intersection remain constant (cf. $\left[Z_{5}\right]$ ),

(11) $X$ is equisingular along $\sigma(D)$ in the sense of Zariski meaning that there exists a projection $\pi: X \rightarrow \mathbb{C} \times D$, such that $f=p r_{2} \circ \pi$ and the discriminant $\Delta_{\pi}$ is of constant multiplicity along $\{0\} \times D \subset \Delta_{\pi}$. (cf. 6.2 and $\left[\mathrm{Z}_{5}\right]$ ).

Nowadays there are several proofs of this theorem and we will make only some comments:

(i) For a plane curve singularity the Puiseux pairs of the branches and their respective intersection multiplicities are determined by the link of the singularity. This follows from results of O. Zariski, K. Brauner and W. Burau. The equivalences

$$
(1) \Leftrightarrow(2) \Leftrightarrow(5) \Leftrightarrow(7) \Leftrightarrow(10)
$$

are then established if one knows that " $\mu_{t}=\mu_{0}$ " implies the invariance of the link in the family. This result was proved by Lê Dũng Tràng [Lê $\hat{1}_{1}$ (see also [L-R]).

(ii) The starting point in Zariski's theory of equisingularity was the property (11) which will be investigated in more detail in 6.2. There it is shown that the equivalence of (7) and (11) remains true for curves which are complete intersections. In general this equivalence fails as is shown by the example (7.2.4).

(ii) O. Zariski $\left(\left[Z_{1}, Z_{5}\right]\right)$ showed that $(10)$ and (11) are equivalent and he introduced the concept of equisaturation which was pursued by $F$. Pham and B. Teissier. Furthermore B. Teissier $\left[\mathrm{Te}_{3}\right]$ gave algebraic proofs for the equivalences

$$
(1) \Leftrightarrow(2) \Leftrightarrow(4) \Leftrightarrow(7) \Leftrightarrow(9) \text {. }
$$

(iv) In the case of plane curves one can even weaken the hypotheses: If $f: X \rightarrow D$ is a (flat) family of plane curve singularities such that $\mu\left(X_{t}\right)$ remains constant then it follows already from results of Cevdet Has Bey and Lazzeri that there is a section of $f$ such that $\sigma(t)$ is the only singularity of $X_{t}$. This is no longer true in the general case (7.2.5).

Up to now the situation for general reduced curve singularities is as follows. Beside the equivalences of 5.2.2 it is shown in [B-G-G] that (7) and (8) are equivalent and that (9) is definitely stronger than these. Furthermore the implication from (7) to (1) is strict (7.2.1).

A particular consequence of the equivalence of (1), (7) and (10) in 5.3.1 is that a deformation of a plane curve singularity $\left(X_{0}, x_{0}\right)$ satisfying one of these 
equivalent conditions induces deformations of each branch of $X_{0}$ at $x_{0}$. These deformations in turn satisfy the equivalent conditions of 5.3.1 too. In the general case this is no longer true as is shown by the counterexamples 7.2.2 and 7.2.3.

\section{Other Numerical Invariants}

We apply the methods developed in Chap. 2 and 3 to investigate the behaviour of several numerical invariants in flat families of curves. We are particularly interested in the torsion part of the holomorphic differential forms $\Omega_{X_{0} . x_{0}}^{1}$ on the reduced curve $\left(X_{0}, x_{0}\right)$. It is well known that the torsion submodule $T\left(\Omega_{X_{0} . x_{0}}^{1}\right)$ contains much information about the singularity itself. In particular, maximal torsion characterizes quasi-homogeneous plane curves $\left[Z_{4}\right]$. On the other hand there is a still unsolved conjecture of Berger which says that the torsion is zero only if $\left(X_{0}, x_{0}\right)$ is smooth. This conjecture is closely related to a conjecture concerning deformation theory of $\left(X_{0}, x_{0}\right)$ because of the well known connection between $T\left(\Omega_{X_{0}, x_{0}}^{1}\right)$ and the Zariski tangent space $T_{X_{0}, x_{0}}^{1}$ of the parameter space of the semi-universal deformation of $\left(X_{0}, x_{0}\right) . T_{X_{0}, x_{0}}^{1}=0$ means that $\left(X_{0}, x_{0}\right)$ is rigid, i.e. allows only trivial deformations, and it is conjectured that there are no rigid curve singularities. For Gorenstein curves this conjecture is equivalent to Berger's conjecture, since then $T_{X_{0}, x_{0}}^{1} \cong T\left(\Omega_{X_{0}, x_{0}}^{1}\right)$ by local duality. But even in this case the answer is in general unknown.

For non-Gorenstein curves, however, the connection between deformations of $\left(X_{0}, x_{0}\right)$ and $T\left(\Omega_{X_{0} . x_{0}}^{1}\right)$ is not obvious. Nevertheless we can show that Berger's conjecture is true for curves which have deformations which lower $\delta$ sufficiently (6.1.4), generalizing results of Pinkham and Bassein. It is also interesting to note that in some cases, smoothable curves have a smooth semiuniversal parameter space if $\Omega_{X_{0}, x_{0}}^{1}$ has minimal torsion (6.1.5).

Section 6.2 is devoted to a generalization of Zariski's discriminant criterion for equisingularity. We succeed in deriving such a criterion only for complete intersection curves. In general we have several possibilities to define invariants of a generic projection. All these invariants coincide for complete intersections, but it is not clear what should be the "correct" one in the general case.

\subsection{The Torsion of the Module of Differentials}

Let $\left(X_{0}, x_{0}\right)$ be a reduced curve singularity, $n:\left(\overline{X_{0}, x_{0}}\right) \rightarrow\left(X_{0}, x_{0}\right)$ the normalization and $c_{X_{0}}: \Omega_{X_{0}, x_{0}}^{1} \rightarrow \omega_{X_{0}, x_{0}}$ the mapping defined in 1.1. $T(M)$ denotes the torsion submodule for any $\mathcal{O}_{X_{0}, x_{0}}$-module $M$.

Definition 6.1.1.

(1) $\tau=\tau\left(X_{0}, x_{0}\right)=\operatorname{dim}_{\mathbb{C}} T\left(\Omega_{X_{0}, x_{0}}^{1}\right)$,

(2) $\bar{\tau}=\bar{\tau}\left(X_{0}, x_{0}\right)=\operatorname{dim}_{\mathbb{C}} T\left(\Omega_{X_{0}, x_{0}}^{1} \otimes_{\mathbb{C}_{X_{0}}, x_{0}} n_{*} \mathcal{O}_{\overline{X_{0}, x_{0}}}\right)$,

(3) $\lambda=\lambda\left(X_{0}, x_{0}\right)=\operatorname{dim}_{\mathbb{C}} \omega_{X_{0}, x_{0}} / c_{X_{0}}\left(\Omega_{X_{0}, x_{0}}^{1}\right)$,

(4) $\kappa=\kappa\left(X_{0}, x_{0}\right)=\operatorname{dim}_{\mathbb{C}} n_{*}\left(\Omega_{\left(X_{0}, x_{0}\right) /\left(X_{0}, x_{0}\right)}^{1}\right)$. 
Note that $\kappa$ is the "cusp-multiplicity" which occurs in the Plücker formulas. $\Omega_{\left(\overline{X_{0}, x_{0}}\right) /\left(X_{0}, x_{0}\right)}^{1}$ denotes the module of relative differential forms.

We shall compare these invariants with

$$
\begin{gathered}
\mu=\mu\left(X_{0}, x_{0}\right)=\operatorname{dim}_{\mathbb{C}} \omega_{X_{0}, x_{0}} / d \mathcal{O}_{X_{0}, x_{0}} \quad \text { (Milnor number), } \\
\delta=\delta\left(X_{0}, x_{0}\right)=\operatorname{dim}_{\mathbb{C}} n_{*} \mathcal{O}_{\left.\overline{X_{0}, x_{0}}\right)} / \mathcal{O}_{X_{0}, x_{0}} \quad(\delta \text {-invariant }), \\
r=r\left(X_{0}, x_{0}\right)=\# \text { irreducible components of }\left(X_{0}, x_{0}\right), \\
m=m\left(X_{0}, x_{0}\right)=\operatorname{dim}_{\mathbb{C}} n_{*} \mathcal{O}_{\left(\overline{X_{0}, x_{0}}\right)} / \mathfrak{M} n_{*} \mathcal{O}_{\overline{\left(X_{0}, x_{0}\right)}} \quad \text { (multiplicity), } \\
\left(\mathfrak{M}=\text { maximal ideal of } \mathfrak{O}_{X_{0}, x_{0}}\right) .
\end{gathered}
$$

Moreover, let $\alpha$ be any numeric, analytical invariant of a reduced curve singularity, and let $f: X \rightarrow T$ be a flat family of reduced curves. Then we set

$$
\begin{gathered}
\alpha_{t}=\alpha\left(X_{t}\right)=\sum_{x \in S\left(X_{t}\right)} \alpha\left(X_{t}, x\right), \\
\alpha_{t}^{\prime}=\alpha^{\prime}\left(X_{t}\right)=\sum_{x \in S\left(X_{t}\right)}\left(\alpha\left(X_{t}, x\right)-1\right),
\end{gathered}
$$

where $S\left(X_{t}\right)$ is the set of singular points of the curve $X_{t}=f^{-1}(t), t \in T$.

Lemma 6.1.2. Let $\left(X_{0}, x_{0}\right)$ be a reduced curve singularity. Then:

(1) $k=m-r$,

(2) $\mu \geqq \lambda \geqq \delta+\kappa$

(3) If $f: X \rightarrow D$ is a small representative of a deformation of $\left(X_{0}, x_{0}\right)$, then $\lambda_{0} \geqq \lambda_{t}$.

Proof. Consider the commutative diagram:

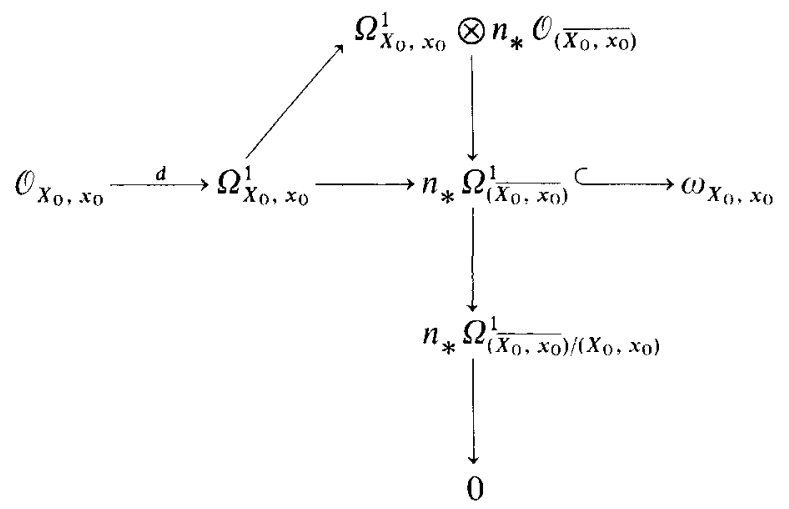

The vertical row is exact, which proves (1). The horizontal line shows that $\mu \geqq \lambda$, and moreover

$$
\begin{aligned}
\lambda & \geqq \operatorname{dim}_{\mathbb{C}} \omega_{X_{0}, x_{0}} / \Omega_{X_{0}, x_{0}}^{1} \otimes n_{*} \mathcal{O}_{\left(\overline{\left.X_{0}, x_{0}\right)}\right.} \\
& =\operatorname{dim}_{\mathbb{C}} \omega_{X_{0}, x_{0}} / n_{*} \Omega_{\left(X_{0}, x_{0}\right)}^{1}+\operatorname{dim}_{\mathbb{C}} n_{*} \Omega_{\left(X_{0}, x_{0}\right)}^{1} / \Omega_{\left(\overline{\left.X_{0}, x_{0}\right)}\right.}^{1} \otimes n_{*} \mathcal{O}_{\left(\overline{\left.X_{0}, x_{0}\right)}\right.} \\
& =\delta+\kappa \quad \text { (by duality (cf. 1.2) and the vertical row). }
\end{aligned}
$$


(3) The sheaf $A=\omega_{X / D} / c_{X / D}\left(\Omega_{X / D}^{1}\right)$ (cf. 4.2.1) is concentrated on the critical set of $f$ which is finite over $D$. Therefore $f_{*} A$ is a coherent $\theta_{D}$-module. Since for all $t \in D$,

$$
\left(f_{*} A\right)(t)=\omega_{X_{t}} / c_{X_{i}}\left(\Omega_{X_{l}}^{1}\right) \quad(\text { cf. } 4.1 .3,4.2 .1)
$$

we obtain $\lambda_{0}=\operatorname{dim}_{\mathbb{C}}\left(f_{*} A\right)(0) \geqq \operatorname{dim}_{\mathbb{C}}\left(f_{*} A\right)(t)=\lambda_{\text {, }}$ (with the notations used in 3.2.3).

Theorem 6.1.3. Let $f: X \rightarrow D$ be a small representative of a germ $f:\left(X, x_{0}\right) \rightarrow(D, 0)$ of a flat family of reduced curves. Then:

(1) $\tau_{0}-\tau_{t}=\lambda_{0}-\lambda_{t}+h_{x_{0}, x_{0}}^{0}\left(\Omega_{X_{i} D}^{1}\right)$.

(2) If, moreover, $f$ is a $\delta$-constant family (4.1.6), then:

$$
\bar{\tau}_{0}-\bar{\tau}_{t}=\left(\mu_{0}+m_{0}^{\prime}\right)-\left(\mu_{t}+m_{t}^{\prime}\right)+h_{x_{0}, X_{0}}^{0}\left(\Omega_{X i D}^{1} \otimes_{c_{X}} n_{*} \mathcal{C}_{\bar{X}}\right) .
$$

Here as always $n: \tilde{X} \rightarrow X$ denotes the normalization of $X$; for the definition of $h_{x_{0}}^{0}, x_{0}$ see 3.2.3. Note that $h_{x_{0}, X_{0}}^{0}\left(\Omega_{X / D}^{1}\right)=h_{x_{0}, X_{0}}^{0}\left(\Omega_{X}^{1}\right)$, and this term vanishes iff $\Omega_{X, x_{0}}^{1}$ contains no elements which are annihilated by some power of $f$. This follows from the exact sequence of local cohomology associated to $0 \rightarrow d f \wedge \mathcal{O}_{X} \rightarrow \Omega_{X}^{1} \rightarrow \Omega_{X}^{1} \rightarrow 0$ and the isomorphism $d f \wedge \mathscr{C}_{X} \cong \mathscr{O}_{X}$.

Corollary 6.1.4. (1) $\tau_{0}-\tau_{t} \geqq \delta_{0}+\kappa_{0}-\mu_{t} \geqq \delta_{0}-2 \delta_{t}+\kappa_{0}$. In particular $T\left(\Omega_{X_{0}, x_{0}}^{1}\right) \neq 0$ if there exists a (flat) deformation of $\left(X_{0}, x_{0}\right)$ such that the generic fiber $X_{t}$ has the property $2 \delta\left(X_{t}\right)<\delta\left(X_{0}, x_{0}\right)$.

(2) If $\left(X_{0}, x_{0}\right)$ is smoothable, i.e. there exists a deformation of $\left(X_{0}, x_{0}\right)$ with generic smooth fiber, then

$$
\left.\tau\left(X_{0}, x_{0}\right) \geqq \lambda\left(X_{0}, x_{0}\right), \quad \text { (Bassein }[\mathrm{Ba}]\right) .
$$

Proof. (1) follows from 6.1.3(1) and 6.1.2(2).

(2) If $X_{t}$ is smooth, then $\tau_{t}=\lambda_{i}=0$.

Remark 6.1.5. In order to improve 6.1.4(1) one might ask if instead of 6.1.2(3) the stronger inequality $\lambda_{0}-\lambda_{t} \geqq \delta_{0}-\delta_{t}$ holds. One may also ask, as in [Ba], if the inequality $\tau \geqq \lambda$ always holds, which would of course imply Berger's conjecture. (We checked it for certain homogeneous, non-smoothable curves.) We pose the question if at least for smoothable curve singularities " $\tau=\lambda$ " always implies that $\left(X_{0}, x_{0}\right)$ is not obstructed (i.e. the base space of the semi-universal deformation of $\left(X_{0}, x_{0}\right)$ is smooth). This was shown by Pinkham [Pi] for smoothable Gorenstein curves.

Proof of 6.1.3. (1) Consider the truncated Poincaré complex

$$
0 \rightarrow \mathcal{O}_{X} \stackrel{d}{\longrightarrow} \Omega_{X / D}^{1} \rightarrow 0,
$$

which satisfies all conditions $\left(\mathrm{P}_{1}\right), \ldots,\left(\mathrm{P}_{5}\right)$. By 3.2.4(3) and 4.2.4(2) we obtain

$$
\operatorname{dim}_{\mathbb{C}} \Omega_{X_{0}, x_{0}}^{1} / d \mathcal{O}_{X_{0}, x_{0}}-\sum_{x \in S\left(X_{t}\right)} \operatorname{dim}_{\mathbb{C}} \Omega_{X_{t}, x}^{1} / d \mathcal{O}_{X_{t}, x}=\mu_{0}-\mu_{t}+h_{x_{0}, X_{0}}^{0}\left(\Omega_{X / D}^{1}\right)
$$


It is easy to see that $d \mathcal{O}_{X_{0}, x_{0}} \cap T\left(\Omega_{X_{0}, x_{0}}^{1}\right)=0$. Therefore we have an exact sequence

$$
0 \rightarrow T\left(\Omega_{X_{0}, x_{0}}^{1}\right) \rightarrow \Omega_{X_{0}, x_{0}}^{1} / d \mathscr{C}_{X_{0}, x_{0}} \stackrel{{ }^{c x_{0}}}{\longrightarrow} \omega_{X_{0}, x_{0}} / d \mathcal{O}_{X_{0}, x_{0}} \rightarrow \omega_{X_{0}, x_{0}} / c_{X_{0}}\left(\Omega_{X_{0}, x_{0}}^{1}\right) \rightarrow 0
$$

and we conclude

$$
\operatorname{dim}_{\mathbb{C}} \Omega_{X_{0}, x_{0}}^{1} / d \mathscr{O}_{X_{0}, x_{0}}=\tau_{0}+\mu_{0}-\lambda_{0} .
$$

The same formula holds for $X_{t}$. Substituting this in $(*)$ we obtain

$$
\mu_{0}-\mu_{t}+h_{x_{0}, X_{0}}^{0}\left(\Omega_{X / D}^{1}\right)=\left(\tau_{0}-\tau_{t}\right)+\left(\mu_{0}-\mu_{t}\right)-\left(\lambda_{0}-\lambda_{t}\right)
$$

and hence the desired result.

(2) We consider the complex

$$
0 \rightarrow \mathcal{O}_{X} \stackrel{d}{\longrightarrow} n_{*} n^{*} \Omega_{X / D}^{1} \rightarrow 0,
$$

where $d$ is the composition of the exterior derivation $\mathcal{O}_{X} \rightarrow \Omega_{X / D}^{1}$ with the canonical mapping $\Omega_{X / D}^{1} \rightarrow n_{*} n^{*} \Omega_{X / D}^{1}=\Omega_{X / D}^{1} \otimes_{C_{X}} n_{*} \mathcal{O}_{\tilde{X}}$. Arguing as before, we obtain

$$
\begin{aligned}
\operatorname{dim}_{\mathbb{W}} & \left(\Omega_{X_{0}, x_{0}}^{1} \otimes n_{*} \mathcal{O}_{\left.\overline{X_{0}, x_{0}}\right)} / d \mathcal{O}_{X_{0}, x_{0}}\right) \\
& =\bar{\tau}_{0}+\operatorname{dim}_{\mathbb{C}} \omega_{X_{0}, x_{0}} / d \mathcal{O}_{X_{0}, x_{U}}-\operatorname{dim}_{\mathbb{C}} \omega_{X_{0}, x_{0}} / \Omega_{X_{0}, x_{0}}^{1} \otimes n_{*} \mathbb{U}_{\left(\overline{X_{0}}, x_{(1)}\right)} \\
& =\bar{\tau}_{0}+\mu_{0}-\delta_{0}-\kappa_{0} .
\end{aligned}
$$

Note that $n_{*} n^{*} \Omega_{X / \boldsymbol{D}, x_{0}}^{1} \otimes \mathcal{O}_{X_{0}, x_{0}}=\Omega_{X_{0}, x_{0}}^{1} \otimes n_{*} \mathcal{O}_{\left(\overline{X_{0}, x_{0}}\right)}$, since $X \rightarrow D$ is supposed to be a $\delta$-constant family and therefore $\tilde{X}_{0}=\bar{X}_{0}$.

Doing the same for $X_{t}$ and substituting in a formula analogous to (*) gives

$$
\bar{\tau}_{0}-\bar{\tau}_{t}=\left(\delta_{0}+\kappa_{0}\right)-\left(\delta_{t}+\kappa_{t}\right)+h_{x_{0}, X_{0}}^{0}\left(n_{*} n^{*} \Omega_{X / D}^{1}\right) .
$$

But since $\delta_{0}=\delta_{t}$, we obtain

$$
\begin{aligned}
\left(\delta_{0}+\kappa_{0}\right)-\left(\delta_{t}+\kappa_{t}\right) & =\left(2 \delta_{0}+\kappa_{0}\right)-\left(2 \delta_{t}+\kappa_{t}\right) \\
& =\left(2 \delta_{0}-r_{0}^{\prime}+m_{0}^{\prime}\right)-\left(2 \delta_{t}-r_{t}^{\prime}+m_{t}^{\prime}\right) \\
& =\left(\mu_{0}+m_{0}^{\prime}\right)-\left(\mu_{t}+m_{t}^{\prime}\right) .
\end{aligned}
$$

From the proof of 6.1.3(1) we obtain the following

Corollary 6.1.6. Let $\left(X_{0}, x_{0}\right)$ be a reduced curve singularity. Then $\tau\left(X_{0}, x_{0}\right)$ $=\lambda\left(X_{0}, x_{0}\right)$ if and only if

$$
\mu\left(X_{0}, x_{0}\right)=\operatorname{dim}_{\mathbb{C}} \Omega_{X_{0}, x_{0}}^{1} / d \mathcal{O}_{X_{0}, x_{0}} .
$$

Since complete intersections are Gorenstein and moreover smoothable and not obstructed, we see by 6.1.5(2) that 1.1.2 is a special case of 6.1.6.

From the results shown above we deduce easily the following semicontinuity theorem. Recall that a function $\alpha: t \mapsto \alpha_{t}$ defined on the complex space $T$ is called upper (resp. lower) semicontinuous is for all $t \in T, \alpha_{t} \geqq \alpha_{t^{\prime}}\left(\operatorname{resp} . \alpha_{t} \leqq \alpha_{t^{\prime}}\right)$ for all $t^{\prime}$ sufficiently near to $t$. 
Theorem 6.1.7. Let $f: X \rightarrow T$ be any flat, analytic family of reduced complex curves.

(1) The following invariants are upper semicontinuous on $T$ :

(a) $\tau, \lambda, \tau-\lambda$,

(b) $\mu, \delta, \mu-\delta$.

(2) If $f$ is a $\delta$-constant family (i.e. $\delta_{t}=$ constant on $T$ ), then the following invariants are upper semicontinuous on $T$ :

(c) $\kappa$

(d) $\bar{\tau}, \mu+m^{\prime}, \bar{\tau}-\left(\mu+m^{\prime}\right)$.

Futhermore, in this case $r^{\prime}$ is lower semicontinuous on $T$.

Proof. It is of course sufficient to prove the theorem in the neighbourhood of each singularity in the fibers of $\mathrm{f}$ and, after base change, for families over a small disc. Then (a) is 6.1.2(3) and 6.1.3(1), (b) is 4.1.4 and 4.2.2.

(c) follows, since for a $\delta$-constant family (4.1.6) $\kappa_{z}=\operatorname{dim}_{\mathbb{C}}\left(f_{*} n_{*} \Omega_{\bar{X} / \mathbb{X}}^{1}\right)(t)$, and $f_{*} n_{*} \Omega_{\overline{\mathrm{x}} / X}^{1}$ is coherent. (d) follows from 6.1.3(2) and $\left(\mu_{0}+m_{0}^{\prime}\right)-\left(\mu_{t}+m_{t}^{\prime}\right)=\kappa_{0}-\kappa_{t}$ if $\delta_{0}=\delta_{t}$ (see the proof of 6.1.3). Furthermore, $r_{t}^{\prime}-r_{0}^{\prime}=\mu_{0}-\mu_{t}$ by 1.2.1(1).

Remarks. (1) By (d) we see that $m^{\prime}$ is upper semicontinuous on $T$, if $f$ is a $\mu$ constant family. For plane curves " $\mu=$ constant" implies even " $m^{\prime}=$ constant", but this is not true in general (cf. example 7.2.1).

(2) In general $r^{\prime}$ and $m^{\prime}$ are in no direction semicontinuous on the base (though the multiplicity is upper semicontinuous on the total space $X$ ). For $r^{\prime}$ this can be seen from the deformation of the cusp into the double point and then smoothing this double point. For $m^{\prime}$ see the following example (cf. [Te $\left.{ }_{4}\right]$, p. 219).

Example 6.1.8. Let $X_{t}$ be the family of plane curves defined by the equation $f(x, y, t)=\prod_{i=1}^{r}\left(y-\alpha_{i}(x+t)(x-t)\right), r \geqq 2$, where $\alpha_{i}$ are pairwise distinct complex numbers. $X_{0}$ has one singular point in 0 and $X_{t}(t \neq 0)$ two singular points in $( \pm t, 0)$. We see immediately: $m\left(X_{0}, 0\right)=m\left(X_{t},( \pm t, 0)\right)=r, r\left(X_{0}, 0\right)=r\left(X_{t},( \pm t, 0)\right)$ $=r$. So $m_{0}^{\prime}=r_{0}^{\prime}=r-1<r_{t}^{\prime}=m_{t}^{\prime}=2(r-1)$. $X_{t}$ is even a $\delta$-constant family with $\delta_{0}$ $=\delta_{\mathrm{t}}=r(r-1)$ (cf. Fig. 2). $r=2$ :
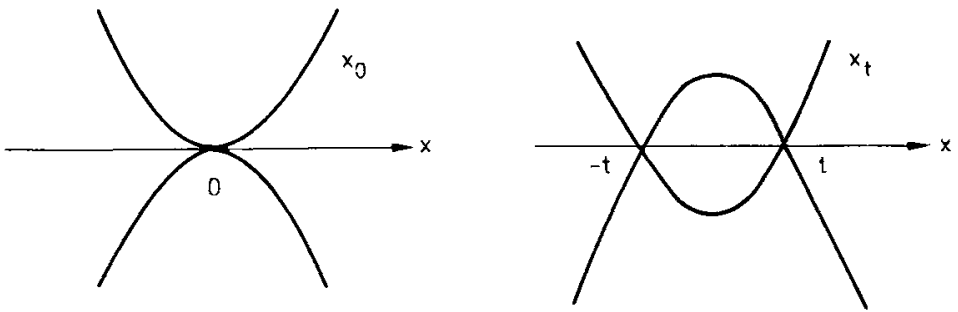

Fig. 2

\subsection{On Zariski's Discriminant Criterion}

Let $\left(X_{0}, x_{0}\right)$ be a reduced curve singularity and $\pi_{L}:\left(X_{0}, x_{0}\right) \rightarrow(L, 0)$ a finite projection on a germ of a complex line. Let $\pi_{L}: X_{0} \rightarrow L$ be a small representative 
which is ramified only in $x_{0}$. The critical set $C_{L}$ (resp. the discriminant $D_{L}$ ) of $\pi_{L}$ consists then only of the point $\left\{x_{0}\right\}$ (resp. $\{0\}$ ) and we put an analytic structure on it by the following definition:

Definition 6.2.1. Let $\pi: X \rightarrow S$ be a finite, flat morphism of complex spaces. We define the critical subspace $C \subset X$, resp. the discriminant subspace $D \subset S$ by

$$
\mathcal{O}_{C}=\mathscr{O}_{X} / F_{0}\left(\Omega_{X / S}^{1}\right), \quad \text { resp. } \mathscr{O}_{D}=\mathcal{O}_{S} / F_{0}\left(\pi_{*} \mathcal{O}_{C}\right)
$$

We call the degree of $\pi$ in $x \in X$ the number

$$
\operatorname{deg}_{x} \pi=\operatorname{dim}_{\mathbb{C}} \mathcal{O}_{\pi^{-1}\{\pi(x), x} .
$$

Here $F_{0}$ denotes the 0 -th Fitting ideal. For an adequate reference concerning discriminants and critical sets we mention Teissier's paper $\left[\mathrm{Te}_{2}\right]$.

We want to compare the multiplicity of $D_{L}$ and other invariants depending on $\pi_{L}$ with invariants of $\left(X_{0}, x_{0}\right)$ itself. Note that $\pi_{L}$, being finite, is automatically flat since $\left(X_{0}, x_{0}\right)$ is Cohen-Macaulay.

For any local ring $\mathcal{O}$ with maximal ideal $\mathfrak{M}$ and any $\mathfrak{M}$-primary ideal $\mathfrak{P}$, $m_{\mathfrak{P}}(\mathcal{O})$ denotes the multiplicity of $\mathfrak{P}$ in $\mathcal{O}$ (in the sense of Samuel and Serre). The multiplicity $m(X, x)$ of a complex germ $(X, x)$ is defined to be the multiplicity of the maximal ideal in $\mathfrak{O}_{X, x}$; for a multigerm the multiplicity is the sum over the local multiplicities.

Definition 6.2.2. Let $\left(X_{0}, x_{0}\right)$ be a reduced curve singularity and $\pi_{L}:\left(X_{0}, x_{0}\right) \rightarrow(L, 0)$ a finite map onto a germ of a complex line. We set:

(1) $\Delta_{L}=A_{L}\left(X_{0}, x_{0}\right)=m\left(D_{L}, 0\right)$

(2) $m_{C_{L}}=m_{C_{L}}\left(X_{0}, x_{0}\right)=m_{\left.F_{0}: \Omega_{X_{0} / L, x_{0}}\right)}\left(\mathcal{O}_{X_{0}, x_{0}}\right)$,

(3) $\tau_{L}=\tau_{L}\left(X_{0}, x_{0}\right)=\operatorname{dim}_{\mathbb{C}} \Omega_{X_{0} / L, x_{0}}^{1}$.

Moreover we write $\operatorname{deg} \pi_{L}$ instead of $\operatorname{deg}_{x_{0}} \pi_{L}$.

Lemma 6.2.3. (1) $\Delta_{L}=m\left(C_{L}, x_{0}\right)=\operatorname{dim}_{\mathbb{C}}\left(\mathcal{O}_{X_{0}, x_{0}} / F_{0}\left(\Omega_{X_{0} / L, x_{0}}^{1}\right)\right)$

(2) $m_{C_{L}}=m\left(n^{*} C_{L}, n^{-1}\left(x_{0}\right)\right)=\operatorname{dim}_{\mathbb{C}}\left(\Omega_{X_{0} / L, x_{0}}^{1} \otimes_{\mathscr{O}_{X_{0}}, x_{0}} n_{*} \mathcal{O}_{\overline{X_{0}, x_{0}}}\right)$

(3) Let $\alpha_{L}$ be one of the three invariants of (6.2.2). Then

$$
\alpha_{L}-\operatorname{deg} \pi_{L}
$$

is independent of the projection $\pi_{L}$.

Proof. Note that the multiplicity of a zero dimensional space is equal to the length of its local ring. Now (1) follows since the matrix defining the Fitting ideal in $\mathscr{O}_{L, 0}$ can be put into diagonal form.

(2) follows from the projection formula and the fact that $\mathcal{O}_{\left(\overline{X_{0}}, x_{0}\right)}$ is principal.

The statement in (3) for $\Delta_{L}=m\left(C_{L}, x_{0}\right)$ and $m_{C L}$ can be proved as follows. One shows that $F_{0}\left(\Omega_{X_{0} / L, x_{0}}^{1}\right) \cdot F_{0}\left(n_{*} \Omega_{\bar{X}_{0} / L, \bar{x}_{0}}^{1}\right)^{-1}$, which is a fractional ideal in the total quotient ring of $\mathcal{O}_{X_{0}, x_{0}}$, is an $\mathcal{O}_{X_{0}, x_{0}}$ submodule of $n_{*} \mathcal{O}_{\left(\overline{X_{0}, x_{0}}\right)}$ and does not depend on $\pi_{L}$. This requires some calculation with the matrix representation of 
$F_{0}\left(\Omega_{X_{0} / L, x_{0}}^{1}\right)$. Then the statement follows from

$$
\begin{aligned}
\operatorname{dim}_{\mathbb{C}} \mathcal{O}_{\left(\overline{\left.X_{0}, x_{0}\right)}\right.} / F_{0}\left(\Omega_{\left(\overline{X_{0}, x_{0}}\right) /(L, 0)}^{1}\right) & =\operatorname{dim}_{\mathbb{C}} F_{0}\left(\Omega_{\left(\overline{X_{0}, x_{0}}\right) /(L, 0)}^{1}\right)^{-1} / \mathcal{O}_{\left(\overline{\left.X_{0}, x_{0}\right)}\right.} \\
& =\operatorname{deg}_{x_{0}} \pi_{L}-r\left(X_{0}, x_{0}\right) .
\end{aligned}
$$

The assertion for $\tau_{L}\left(X_{0}, x_{0}\right)$ is originally due to R. Berger [Be] and contained in the following proposition.

Proposition 6.2.4. With the assumptions and notations of 6.2 .2 and 6.1 .1 the following holds:

(1) $\tau_{L}-\operatorname{deg} \pi_{L}+1=\tau-\hat{\lambda}+\mu$

(2) $m_{C_{L}}-\operatorname{deg} \pi_{L}=\bar{\tau}-m$.

Proof. Consider the following diagram with exact rows,

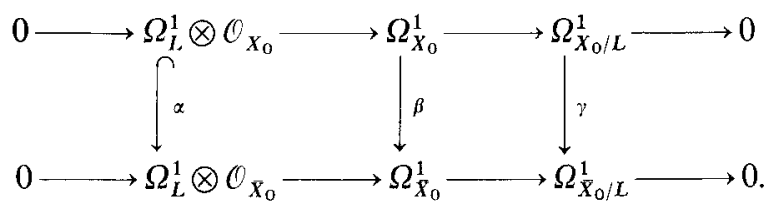

We obtain $\operatorname{ker} \alpha=0, \operatorname{dim}_{\mathbb{C}} \operatorname{coker} \alpha=\delta, \operatorname{dim}_{\mathbb{C}} \operatorname{ker} \beta=\tau, \operatorname{dim}_{\mathbb{C}} \operatorname{coker} \beta=\lambda-\delta$, $\operatorname{dim}_{\mathbb{C}} \Omega_{\left(\overline{X_{0}, x_{0}}\right) /(L, 0)}^{1}=\operatorname{deg} \pi_{L}-r$.

Therefore,

$$
\operatorname{dim}_{\mathbb{C}} \operatorname{ker} \gamma-\operatorname{dim}_{\mathbb{C}} \operatorname{coker} \gamma=\tau+\delta-(\lambda-\delta)
$$

and

$$
\tau_{L}=\operatorname{deg} \pi_{L}-r+(\tau-\lambda)+2 \delta,
$$

which proves (1).

Tensoring the first row of the diagram above with $\mathcal{O}_{\left(\overline{X_{0}, x_{0}}\right)}$ and looking at the resulting diagram, we obtain

$$
\begin{aligned}
\bar{\tau}-\kappa & =\operatorname{dim}_{\mathbb{C}} \Omega_{X_{0}, x_{0}}^{1} \otimes \mathcal{O}_{\left(\overline{X_{0}, x_{0}}\right)}-\operatorname{dim}_{\mathbb{C}} \Omega_{\left(\overline{X_{0}, x_{0}}\right) /(\varepsilon, 0\}}^{1} \\
& =m_{C_{L}}-\operatorname{deg} \pi_{L}+r .
\end{aligned}
$$

But $\kappa=m-r$ by 6.1 .2 , hence the result.

Definition 6.2.5. Let $\alpha_{L}$ be one of the invariants $\Delta_{L}, m_{C_{L}}, \tau_{L}$, of 6.2.2. We define

$$
\alpha_{\pi}=\inf \alpha_{L},
$$

where the infimum is taken over all possible finite maps $\pi_{L}:\left(X_{0}, x_{0}\right) \rightarrow(L, 0)$. If $\pi_{L}$ is such that $\alpha_{L}=\alpha_{\pi}$ we call $\pi_{L}$ a generic projection. The infimum $\alpha_{\pi}$ is already achieved if we consider only finite linear projections in the following sense: Choose a fixed embedding $\left(X_{0}, x_{0}\right) \subset\left(\mathbb{C}^{n}, x_{0}\right)$ and let $L \subset \mathbb{C}^{n}$ be any line through $x_{0}$. A linear projection $\pi_{L}:\left(X_{0}, x_{0}\right) \rightarrow\left(L, x_{0}\right)$ is the restriction to $\left(X_{0}, x_{0}\right)$ of the orthogonal (with respect to some Hermitian metric) projection $\mathbb{C}^{n} \rightarrow L$.

Now $\alpha_{L}=\alpha_{\pi}$ for $L$ in that Zariski open subset of $\mathbb{P}^{n-1}$ where $\operatorname{deg}_{L} \pi$ achieves its minimum $m\left(X_{0}, x_{0}\right)$ by 6.2.3(3). Therefore $\pi_{L}$ is generic (with respect to $A_{L}$, $m_{C_{L}}$ or $\left.\tau_{L}\right)$ iff $\operatorname{deg}_{X_{0}} \pi_{L}=m\left(X_{0}, x_{0}\right)$. 
Remark 6.2.6. (1) If $\left(X_{0}, x_{0}\right)$ is a complete intersection, then $F_{0}\left(\Omega_{X_{0} / L, x_{0}}^{1}\right)$ is a principal ideal and we obtain

$$
\Delta_{L}=m_{C_{L}}=\tau_{L}=\mu+\operatorname{deg} \pi_{L}-1
$$

by $6.2 .4(1), 6.1 .5(2)$. Note that the formula for $\Delta_{L}$ can be generalized to complete intersections of arbitrary dimensions by $\mu\left(X_{0}, x_{0}\right)+\mu\left(\pi_{L}^{-1}(0), x_{0}\right)$ (cf. $\left[\mathrm{G}_{1}\right]$, Lemma 5.3). For a generic projection $\pi$ we obtain

$$
\Delta_{\pi}=m_{C_{\pi}}=\tau_{\pi}=\bar{\tau}=\mu+m-1 .
$$

(2) Except for the case of complete intersections we do not have an estimate for $A_{\pi}$. But using the results of $[\mathrm{Be}]$ and $\left[\mathrm{K}-\mathrm{H}_{1}\right]$ about the relation between Kähler and Dedekind different, one can deduce the following: If $\left(X_{0}, x_{0}\right)$ is Gorenstein, then

$$
\Delta_{\pi} \geqq \mu+m-1,
$$

and equality holds iff $\left(X_{0}, x_{0}\right)$ is a complete intersection. But one may have $\Delta_{\pi}$ $=\mu+m-1$ for $\left(X_{0}, x_{0}\right)$ not Gorenstein (e.g. the ordinary triple point). Note that always $\tilde{\tau}=m_{C_{\pi}} \geqq \Delta_{\pi}$ (by 6.2.3(1)) and equality holds iff the critical space of the projection is a hypersurface.

The following theorem is a generalization of Zariski's discriminant criterion for plane curves $\left(\left[\mathrm{Z}_{1}\right]\right)$.

Theorem 6.2.7. Let $f:\left(X, x_{0}\right) \rightarrow(D, 0)$ be a (flat) deformation of a reduced complete intersection curve $\left(X_{0}, x_{0}\right)$ and $f: X \rightarrow D$ a sufficiently small representative.

The following conditions are equivalent:

(1) Let $\pi=\left(\pi_{1}, f\right):\left(X, x_{0}\right) \rightarrow(L \times D, 0)$ be any finite generic projection (i.e. $\left.\operatorname{deg}_{x_{0}} \pi=m\left(X_{0}, x_{0}\right)\right)$. Then the reduced discriminant $\left(D_{\pi}\right)_{\mathrm{red}}$ of $\pi$ is smooth.

(2) There exists a finite mapping $\pi=\left(\pi_{1}, f\right):\left(X, x_{0}\right) \rightarrow(L \times D, 0)$ such that the multiplicity $m\left(D_{\pi},(0, t)\right)$ of $D_{\pi}$ along $\{0\} \times D$ is constant for all $t \in D$ and equal to $\mu\left(X_{t}\right)+m^{\prime}\left(X_{t}\right)$ for $t \neq 0$.

(3) $f: X \rightarrow D$ admits a holomorphic section $\sigma: D \rightarrow X$ such that $X_{t}-\sigma(t)$ is smooth and $\mu\left(X_{t}, \sigma(t)\right)$ and $m\left(X_{t}, \sigma(t)\right)$ are constant for $t \in D$.

(4) For each $t \in D$ there exists an $x_{\mathrm{f}} \in X_{t}$ such that $\mu\left(X_{t}, x_{t}\right)+m\left(X_{t}, x_{t}\right)$ is equal to $\mu\left(X_{0}, x_{0}\right)+m\left(X_{0}, x_{0}\right)$.

Before we prove the theorem we need a lemma which is interesting in itself. We set

$$
\pi_{\Sigma_{t}}=\left.\pi\right|_{X_{t}}: X_{t} \rightarrow L \times\{t\}=L_{t} .
$$

Because all fibers $X_{t}$ are complete intersections we obtain by $6.2 .6(1)$

$$
\Delta_{L_{t}}\left(X_{t}, x\right)=\mu\left(X_{t}, x\right)+\operatorname{deg}_{x} \pi_{L_{t}}-1, \quad x \in X_{t} .
$$

We set

$$
v_{L_{t}}\left(X_{t}, x\right)=\operatorname{deg}_{x} \pi_{L_{t}}-m\left(X_{t}, x\right), \quad x \in X_{t},
$$

and call it the number of vertical tangents of $\pi_{L_{t}}$ in $x$. Note that $\pi:\left(X, x_{0}\right) \rightarrow$ $(L \times D, 0)$ is generic iff $v_{L_{0}}\left(X_{0}, x_{0}\right)=0$. 
Moreover we set

$$
v_{L_{t}}\left(X_{i}\right)=\sum_{x \in X_{i}} v_{L_{i}}\left(X_{i}, x\right)
$$

Lemma 6.2.8. With the assumptions and notations of 6.2 .7 , let $\pi=\left(\pi_{1}, f\right)$ : $\left(X, x_{0}\right) \rightarrow(L \times D, 0)$ be a finite mapping. Then

$$
\mu_{0}-\mu_{t}=\left(m_{t}^{\prime}-m_{0}^{\prime}\right)+\left(v_{L_{t}}\left(X_{i}\right)-v_{L_{0}}\left(X_{0}, x_{0}\right)\right)
$$

In particular, $\mu+m^{\prime}$ is upper semicontinuous on $D$.

Proof. $D_{\pi}$ is a hypersurface since $\left(X, x_{0}\right)$ is a complete intersection. Therefore the first statement is just

$$
\Delta_{L_{0}}\left(X_{0}, x_{0}\right)=i\left(L_{0} \cdot D_{\pi}, 0\right)=i\left(L_{t} \cdot D_{\pi}\right)=\sum_{\substack{s \in L_{t} \cap D_{\pi} \\ x \in \pi^{-1}(s, t)}} \Delta_{L_{t}}\left(X_{t}, x\right),
$$

where $i$ denotes the intersection multiplicity, together with the formulas for $\Delta_{L_{t}}$ and the definition of $v_{L_{t}}$. Choosing $\pi$ generic, the second statement follows.

Remark. Suppose that $f$ is a $\mu$-constant family. We have already noticed that $m^{\prime}$ is upper semicontinuous (Remark (1) after 6.1.7). For complete intersections we see that $m_{0}^{\prime}-m_{t}^{\prime}$ is just the number of vertical tangents of $X_{t}$ for a generic projection.

Proof of 6.2 .7 (a) Let us first assume that $\pi=\left(\pi_{1}, f\right):\left(X, x_{0}\right) \rightarrow(L \times D, 0)$ is any finite projection such that $\left(D_{\pi}\right)_{\text {red }}$ is smooth. We show that this implies (3) except that the multiplicity $m\left(X_{t}, \sigma(t)\right)$ might not be constant. Note that $D_{\pi}$ is a hypersurface since $\left(X, x_{0}\right)$ is a complete intersection.

W.1.o.g. we may assume that $\left(D_{\pi}\right)_{\mathrm{red}}=\{0\} \times D$. Since $m\left(D_{\pi},(0, t)\right)$ $=i\left(L_{t} \cdot D_{\pi},(0, t)\right)$ is constant for all $t$, we obtain:

$$
\begin{aligned}
\mu_{0}+\operatorname{deg}_{x_{0}} \pi_{L_{0}}-1 & =\Delta_{L_{0}}\left(X_{0}, x_{0}\right)=\sum_{x \in \pi^{-1}(0, t)} \Delta_{L_{t}}\left(X_{i}, x\right) \\
& =\mu_{t}+\sum_{x \in \pi^{-1}(0,1)}\left(\operatorname{deg}_{x} \pi_{L_{t}}-1\right) \\
& =\mu_{t}+\operatorname{deg}_{x_{0}} \pi_{L_{0}}-\#\left\{\pi^{-1}(0, t)\right\},
\end{aligned}
$$

because $\pi$ is flat. Since $\mu$ is upper semicontinuous we obtain $\mu_{0}=\mu_{t}$ and $\#\left\{\pi^{-1}(0, t)\right\}=1$. In particular

$$
\pi:\left(C_{\pi}\right)_{\mathrm{red}} \rightarrow\left(D_{\pi}\right)_{\mathrm{red}}=\{0\} \times D
$$

is unramified and $1-1$, hence biholomorphic. The inverse gives a section $\sigma$, and $X_{t}-\sigma(t)$ is smooth.

(b) $(1) \Rightarrow(2)$. Let $\pi$ be any generic projection. By hypothesis and by (a) we get

$$
m\left(D_{\pi},(0, t)\right)=\mu_{t}+\operatorname{deg}_{\sigma(t)} \pi_{L_{t}}-1 \geqq \mu_{t}+m_{t}-1 .
$$

But after a small change of $\pi$, which again is generic, we may assume that $\operatorname{deg}_{\sigma(t)} \pi_{L_{t}}=m_{t}$.

$(2) \Rightarrow(3)$. By (a) we have only to show that $m\left(X_{t}, \sigma(t)\right)$ remains constant, since the hypothesis implies that $\left(D_{\pi}\right)_{\text {red }}$ is smooth. 
But $\mu_{0}+m_{0}-1 \leqq m\left(D_{\pi},(0,0)\right)=m\left(D_{\pi},(0, t)\right)=\mu_{t}+m_{t}-1$ and $\mu_{0}+m_{0} \geqq \mu_{t}+m_{t}$ by 6.2.8. Hence $m_{0}=m_{t}$ since $\mu$ is constant.

Since $(3) \Rightarrow(4)$ is trivial, we have to show $(4) \Rightarrow(1)$. Consider any finite projection $\pi:\left(X, x_{0}\right) \rightarrow(L \times D, 0)$. By 6.2 .8 we obtain

$$
\begin{aligned}
\mu_{0}+m_{0}^{\prime} & =\mu_{t}+m_{t}^{\prime}+v_{L_{t}}\left(X_{t}\right) \\
& \geqq \mu\left(X_{t}, x_{t}\right)+m^{\prime}\left(X_{t}, x_{t}\right)+v_{L_{t}}\left(X_{t}\right) .
\end{aligned}
$$

Using the assumption, we conclude that $X_{t}-\left\{x_{t}\right\}$ must be smooth and that $i\left(L_{t} \cdot D_{\pi}, \pi\left(x_{t}\right)\right)$ is constant. Hence $\left(D_{\pi}\right)_{\text {red }}$ must be smooth.

\section{Applications and Examples}

So far we have proved properties of families of curves in analogy with the case of plane curves. Now we are going to discuss several examples which show the essential differences.

To do this we need a simple criterion to decide whether a given family $\left(X_{t}\right)_{t \in D}$ is a deformation of $X_{0}$. If $X_{0}$ is given by equations $f_{1}(x), \ldots, f_{l}(x), x \in \mathbb{C}^{n}$, and $X_{t}$ is defined by a "deformation of the equations", $F_{i}(x, t)=f_{i}(x)+t g_{i}(x, t), t \in D$, then it is in general difficult to check whether this family is flat. One has to show that every relation between the $f_{i}$ can be lifted to a relation between the $F_{i}$. But if $X_{0}$ is given by a parametrization $\varphi_{0}: \bar{X}_{0} \rightarrow \mathbb{C}^{n}$, and $\left(X_{t}\right)_{t \in D}$ is given by a "deformation of the parametrization", $\varphi: \bar{X}_{0} \times D \rightarrow \mathbb{C}^{n}, \varphi(s, t)=\varphi_{0}(s)+t \psi(s, t)\left(s \in \bar{X}_{0}\right.$, $t \in D)$, that is $X_{t}=\varphi\left(\bar{X}_{0} \times\{t\}\right)$ with its reduced structure, then one has to decide whether this is indeed an analytic family. This is however comparatively easy; a necessary and sufficient condition is that $\delta\left(X_{t}\right)$ is constant.

We end with a discussion of the ordinary $n$-tuple point. The minimality of $\mu$ already allows us to determine all possible deformations. Moreover we show that the monodromy group is trivial.

\section{1. $\delta$-Constant Deformations}

The results of this section are essentially due to B. Teissier $\left(\left[\mathrm{Te}_{2}\right], 3.2\right)$.

Let $\left(X_{0}, x_{0}\right) \subset\left(\mathbb{C}^{n}, 0\right)$ be a germ of a reduced curve and $X_{0} \subset B_{0}$ a representative, where $B_{0} \subset \mathbb{C}^{n}$ is a small open ball with center $x_{0}$. We assume that $X_{0}$ is given by a parametrization, i.e. as the (reduced) image of a holomorphic mapping

$$
\begin{aligned}
\varphi_{0}: \bar{X}_{0} & =\coprod_{j=1}^{r} D_{j} \rightarrow X_{0} \subset B_{0}, \\
\varphi_{0}(s) & =\left(\varphi_{0,1}(s), \ldots, \varphi_{0, n}(s)\right),
\end{aligned}
$$

where $\coprod_{j=1}^{r} D_{j}$ is the disjoint union of $r$ open discs, such that $D_{j}$ is mapped homeomorphically on exactly one branch $X_{0}^{j}$ of $X_{0}$ and $\varphi_{0}(0)=x_{0}, 0 \in D_{j}$, for each $j$. We shall sometimes not distinguish between $\varphi_{0}$ and its (multi-) germ at 
$\bar{x}_{0}=\varphi_{0}^{-1}\left(x_{0}\right)=\left(0_{j}\right\}_{j=1, \ldots, r} \in \prod_{j=1}^{r} D_{j}, \varphi_{0}: \bar{X}_{0} \rightarrow X_{0}$ is the normalization of $X_{0}$ and the local algebra $\mathcal{O}_{X_{0}, x_{0}}$ is the subalgebra of $\varphi_{0} * \mathcal{O}_{\left(\overline{\left.X_{0}, x_{0}\right)}\right.}=\underset{j=1}{r} \mathbb{C}\left\{s_{j}\right\}$ generated by the component functions

of $\varphi_{0}$.

$$
\varphi_{0 . i}(s)=\left(\varphi_{0, i}^{j}\left(s_{j}\right)\right)_{j=1, \ldots, r} \in \bigoplus_{j=1}^{r} \mathbb{C}\left\{s_{j}\right\}
$$

Let $D \subset \mathbb{C}$ be a small disc with center 0 . A deformation of the parametrization is a holomorphic mapping

$$
\varphi: \bar{X}_{0} \times D=\coprod_{j=1}^{r}\left(D_{j} \times D\right) \rightarrow B_{0},
$$

such that $\varphi(s, t)=\varphi_{0}(s)+t \psi(s, t), s \in \bar{X}_{0}, t \in D$.

Then for sufficiently small $D_{j}$ and $D$ the following holds:

(a) $\Phi=(\varphi, t): \bar{X}_{0} \times D \rightarrow B_{0} \times D$ is a finite mapping and therefore

$$
Y=\Phi\left(\bar{X}_{0} \times D\right) \subset B_{0} \times D
$$

is a two dimensional analytic subset which we give the reduced structure. Let

$$
f: Y \rightarrow D
$$

denote the projection on the second factor and set $Y_{t}=f^{-1}(t)$.

(b) For each fixed $t \in D$ define

$$
\varphi_{t}: \bar{X}_{0} \rightarrow B_{0} \quad \text { by } \varphi_{t}(s)=\varphi(s, t)
$$

and set $X_{t}=\varphi_{t}\left(\bar{X}_{0}\right)$ with its reduced structure. Therefore $X_{t}=\left(Y_{t}\right)_{\text {red }}$. Note that $\mathcal{O}_{Y,\left(x_{0}, 0\right)}$ is the subalgebra of $\Phi_{*} \mathcal{O}_{\bar{X}_{0} \times D,\left(x_{0}, 0\right)}=\bigoplus_{j=1}^{r} \mathbb{C}\left\{s_{j}, t\right\}$ generated by the (multi-) germs of $t$ and of the component functions $\varphi_{i}(s, t)$ of $\varphi$ at $\left(\bar{x}_{0}, 0\right) . \mathcal{O}_{Y_{t}}=$ $\mathcal{O}_{Y} /(f-t) \mathcal{O}_{Y}$ while $\mathcal{O}_{X_{t}}$ is the image of $\mathcal{O}_{Y_{\tau}}$ in $\Phi_{*} \mathcal{O}_{X_{0} \times D} /(f-t) \mathcal{O}_{X_{0} \times D}$.

Lemma 7.1.1. (1) $\Phi: \bar{X}_{0} \times D \rightarrow Y$ is the normalization of $Y$. Hence $\varphi_{t}: \bar{X}_{0} \rightarrow X_{t}$ is the normalization of $X_{t}$ for all $t \in D$.

(2) $f: Y \rightarrow D$ is flat, $Y_{0}-\{0\}$ is reduced and $Y_{i}$ is reduced for each $t \neq 0$.

Proof. (1) Let $Y_{i}$ be an irreducible component of $Y$. Using Sard's theorem and Ritt's lemma we see that $\Phi: \Phi^{-1}\left(Y_{i}\right) \rightarrow Y_{i}$ is regular over the complement of some curve $C_{i}$ in $Y_{i}$ and therefore an unramified covering over $Y_{i}-C_{i}$. Since $\varphi_{0}$ is regular in each point of $\bar{X}_{0}-\varphi_{0}^{-1}\left(x_{0}\right)$, so is $\Phi$ and therefore $C_{i} \cap X_{0}=\left\{x_{0}\right\}$. So $Y_{i}$ $-C_{i}$ contains points of $X_{0}$ and each of these points has only one point as preimage. This implies that $\Phi$ is biholomorphic over $Y_{i}-C_{i}$ and (1) follows from the universal property of normalizations.

(2) Since $f$ is not contained in any associated prime ideal of $\mathcal{O}_{Y, x_{0}}, f$ is a nonzero divisor which means that $f$ is flat. $\bar{X}_{0} \times D$ and $Y$ are biholomorphic outside the 
singular set $S(Y)$ of $Y_{0}$. Therefore $Y_{0}-\{0\}$ is reduced and $Y_{t}, t \neq 0$, is reduced except at the finitely many points of $Y_{t} \cap S(Y)$.

Since $Y$ is reduced we may assume that $Y-\left\{x_{0}\right\}$ is Cohen-Macaulay. Since $f$ is flat, $Y_{t}, t \in D-\{0\}$, is Cohen-Macaulay and therefore without embedded components, hence reduced everywhere.

The lemma shows that $f: Y \rightarrow D$ is always a deformation of $Y_{0}=f^{-1}(0)$. It is a deformation of $X_{0}$ iff $Y_{0}$ is reduced.

Deformation 7.1.2. Let $\varphi: \bar{X}_{0} \times D \rightarrow B_{0}$ be a deformation of a parametrization of $X_{0}$. We say that $\varphi$ defines a deformation of $X_{0}$ if $Y_{0}$ is reduced.

The following criterion has been implicitly used already in [Pi].

Proposition 7.1.3 (Teissier). The following conditions are equivalent:

(1) The deformation of the parametrization, $\varphi$, defines a deformation of $X_{0}$.

(2) $\delta\left(X_{t}\right)$ is constant for all $t \in D$.

Proof. Consider the following commutative diagram, resp. isomorphism, resp. exact sequences:

$(\alpha)$

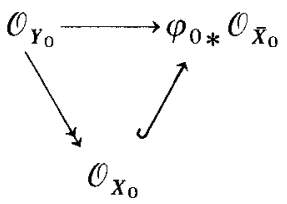

$(\beta)\left(\varphi_{*} \mathcal{O}_{\bar{X}_{0} \times D} / \mathcal{O}_{Y}\right) \otimes_{\mathcal{O}_{Y}} \mathcal{O}_{Y_{t}} \cong \varphi_{0 *} \mathcal{O}_{X_{0}} / \mathcal{O}_{X_{t}}, t \in D$

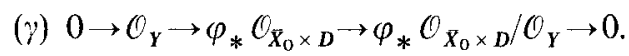

Tensoring with $\otimes_{\mathscr{O}_{Y}} \mathcal{O}_{Y_{0}}$ yields

$$
\mathcal{O}_{Y_{0}} \rightarrow \varphi_{0 *} \mathcal{O}_{\bar{X}_{0}} \rightarrow \varphi_{0 *} \mathcal{O}_{\bar{X}_{0}} / \mathcal{O}_{X_{0}} \rightarrow 0
$$

Because of $(\beta), \delta\left(X_{t}\right)=$ constant is equivalent to $f_{*}\left(\varphi_{*} \mathcal{O}_{\bar{X}_{0 \times D}} / \mathcal{O}_{Y}\right)$ being a locally free $\mathcal{O}_{D}$-module. By $(\gamma)$ this is equivalent to

$$
\mathcal{O}_{Y_{0}} \rightarrow \varphi_{0 *} \mathcal{O}_{X_{0}} \quad \text { being injective }
$$

By $(\alpha)$ this is equivalent to $\mathcal{O}_{Y_{0}} \rightarrow \mathcal{O}_{X_{0}}$ being injective.

Remark 7.1.4. (1) With the notations introduced above, assume that $\left(X_{t}\right)_{t \in D}$ is an analytic family, i.e. there exists a complex space $X$, a holomorphic mapping $g: X \rightarrow D$ and for each $t \in D$ an isomorphism $g^{-1}(t) \cong X_{t}$. It is not very difficult to show that this implies: $X$ is reduced, $g$ is flat (hence a deformation of $X_{0}$ ) and the normalization $\tilde{g}: \tilde{X} \rightarrow D$ is isomorphic to $\bar{X}_{0} \times D \rightarrow D$. Therefore the conditions of 7.1.3 are equivalent to the condition that $\left(X_{t}\right)_{t \in D}$ is an analytic family.

(2) Let $\varphi_{0}$ be a parametrization of $X_{0}$ and $\varphi$ a deformation of $\varphi_{0}$ as before. Consider the (multi-) mapping:

$$
\begin{gathered}
\varphi_{t}: \bar{X}_{0}=\coprod_{j=1}^{r} D_{j} \rightarrow B_{0} \subset \mathbb{C}^{n}, \quad t \in D, \\
\varphi_{t}\left(s_{j}\right)_{j=1, \ldots, r}=\left(\varphi_{t, 1}^{j}\left(s_{j}\right), \ldots, \varphi_{t, n}^{j}\left(s_{j}\right)\right)_{j=1, \ldots, r} .
\end{gathered}
$$


The subsheaf $\mathcal{O}_{X_{t}} \subset \varphi_{t *} \mathcal{O}_{X_{0}} \cong \bigoplus_{j=1}^{r} \mathcal{O}_{D_{j}}$ is generated by the component functions $\varphi_{t, i}$ of $\varphi_{t} . \delta\left(X_{t}\right)$ is the dimension of the quotient sheaf and this quotient is concentrated on those points $x=\left(x_{1}, \ldots, x_{n}\right)$ where one of the following conditions is satisfied:

(a) There exist $s_{j}^{0} \neq s_{k}^{1} \quad\left(s_{j}^{0} \in D_{j}, s_{k}^{1} \in D_{k}\right)$ such that $\varphi_{t, i}^{j}\left(s_{j}^{0}\right)=\varphi_{t, i}^{k}\left(s_{k}^{1}\right)$ for all $i=1, \ldots, n$.

(b) There exists a $s_{j}^{0} \in D_{j}$ such that $\frac{\partial \varphi_{i, i}^{s}}{\partial s_{j}}\left(s_{j}^{0}\right)=0$ for all $i=1, \ldots, n$.

In particular: If $X_{0}$ is irreducible $(r=1), \varphi_{t}(0)=0$ for all $t$ and $\operatorname{dim}_{\mathbb{C}} \mathbb{C}\{s\} /\left(\varphi_{t, 1}, \ldots, \varphi_{t, n}\right)=$ constant, then $\varphi$ defines a deformation of $X_{0}$.

\subsection{Examples and Counterexamples}

To illustrate the difference from the case of plane curves we shall consider families $\left(X_{t}\right)_{t \in D}$ of irreducible curves which can be given by a deformation $\varphi_{1}(s, t), \ldots, \varphi_{n}(s, t) \in \mathbb{C}\{s, t\}$ of the parametrization $\varphi_{1}(s, 0), \ldots, \varphi_{n}(s, 0) \in \mathbb{C}\{s\}$ of $X_{0}$ (cf. the notation of 7.1). We define $\left(X_{t}\right)_{t \in D}$ by the subalgebra $\mathbb{C}\left\{\varphi_{1}(s, t), \ldots, \varphi_{n}(s, t)\right\} \subset \mathbb{C}\{s, t\}$. Recall that $\mu\left(X_{t}\right)=$ constant implies $\delta\left(\mathrm{X}_{t}\right)=$ constant. None of the phenomena of 7.2.1 to 7.2.5 can occur for plane curves.

Example 7.2.1. Constant Milnor number does not imply constant multiplicity:

(a) $\mathbb{C}\left\{s^{3}-s^{2} t, s^{4}, s^{5}\right\}, \mu\left(X_{t}, 0\right)=4$ for all $t$ but $m\left(X_{0}, 0\right)=3, m\left(X_{t}, 0\right)=2$ $(t \neq 0)$.

(b) (J.P. Henry). Define $X_{t} \subset \mathbb{C}^{3}$ by the equations

$$
x y-t z=0, \quad z^{6}+x^{15}+y^{10}=0 .
$$

$X_{t}$ is quasihomogeneous; $\left(X_{0}, 0\right)$ is a complete intersection and $\left(X_{t}, 0\right), t \neq 0$, a plane curve singularity. We find

$$
\mu\left(X_{t}, 0\right)=126(\text { all } t), \quad m\left(X_{0}, 0\right)=12, \quad m\left(X_{r}, 0\right)=10(t \neq 0) .
$$

Example 7.2.2. Constant Milnor number does not imply constant Milnor number of each branch:

In the example of Henry, $X_{t \neq 0}$ has 5 branches with semigroup (cf. $\left[\mathrm{H}-\mathrm{K}_{2}\right]$ ) $\langle 2,3\rangle(\mu=2)$ while $X_{0}$ has 3 branches with semigroup $\langle 2,5\rangle(\mu=4)$ and 2 branches with semigroup $\langle 3,5\rangle(\mu=8)$.

This is due to the following fact. If $f: X \rightarrow D$ denotes the family, then $X$ has 5 irreducible, reduced components $X^{i}$. Let $f^{i}: X^{i} \rightarrow D$ be the restriction of $f$. Of course $\left(f^{i}\right)^{-1}(0)_{\text {red }}$ is equal to some branch of $X_{0}$. But $\left(f^{i}\right)^{-1}(0)$ itself is not reduced; it has an embedded component in 0 .

Example 7.2.3. Constant Milnor number and constant multiplicity do not imply constant semigroup:

$$
\mathbb{C}\left\{s^{4}, s^{7}+t s^{6}, s^{9}, s^{10}\right\}, \mu\left(X_{t}, 0\right)=12, \quad m\left(X_{t}, 0\right)=4 \text { for all } t,
$$

but

$$
\Gamma\left(X_{0}, 0\right)=\langle 4,7,9,10\rangle, \quad \Gamma\left(X_{t \neq 0}, 0\right)=\langle 4,6,9,11\rangle
$$


Example 7.2.4. Constant semigroup does not imply constant multiplicity of the discriminant of a generic line projection (cf. 6.2):

$$
\begin{gathered}
\mathbb{C}\left\{s^{6}, s^{8}+2 t s^{9}, s^{10}+t s^{11}, s^{17}, s^{19}+\frac{2}{3} t s^{21}\right\} \quad\left(\text { cf. }\left[\mathrm{H}-\mathrm{K}_{2}\right] \text { p. } 40\right) . \\
\Gamma\left(X_{t}, 0\right)=\langle 6,8,10,17,19\rangle \quad \text { for all } t .
\end{gathered}
$$

$\left(X_{0}, 0\right)$ is Gorenstein, but not a complete intersection, $\left(X_{t \neq 0}, 0\right)$ is a complete intersection. By $\left(\left[\mathrm{H}-\mathrm{K}_{2}\right]\right)$ we obtain

$$
\Delta_{\pi}\left(X_{0}, 0\right)>\Delta_{\pi}\left(X_{t}, 0\right)=\mu\left(X_{t}, 0\right)+m\left(X_{t}, 0\right)-1=27 \quad(t \neq 0) .
$$

This is due to the following fact: let $f: X \rightarrow D$ denote the family and $\pi: X \rightarrow L$ $\times D$ a generic plane projection (cf. 6.2). Then the reduced discriminant $\left(D_{\pi}\right)_{\mathrm{red}}$ is smooth, but $D_{\pi}$ has an embedded component in 0 .

Example 7.2.5. Constant Milnor number does not imply "non-splitting" of the singularity:

$$
\mathbb{C}\left\{s^{3}-3 t s, s^{4}-2 t s^{2}, s^{5}-7 t s^{3}+16 t^{2} s\right\} .
$$

$X_{t \neq 0}$ has two singular points for $s= \pm \sqrt{t}$, each isomorphic to $\mathbb{C}\left\{t^{2}, t^{3}\right\}$, Hence $\mu\left(X_{0}, 0\right)=\mu\left(X_{t}\right)=4$.

Question. Do there exist examples of type 7.2.1 and 7.2.3 for irreducible complete intersections or of type 7.2 .5 for arbitrary complete intersections?

Deformations of the ordinary multiple point:

Proposition 7.2.6. Let $\left(X_{0}, x_{0}\right) \subset\left(\mathbb{C}^{n}, x_{0}\right)$ be the ordinary n-tuple point (1.2.4). The following holds:

(1) Let $f: X \rightarrow D$ be any small representative of a deformation of $\left(X_{0}, x_{0}\right)$. Then $X_{t}=f^{-1}(t), t \in D$, has only ordinary multiple points as singularities.

(2) Let $n_{1}, \ldots, n_{p}$ be any integers such that $n_{i} \geqq 2$ and $\sum_{i=1}^{p}\left(n_{i}-1\right) \leqq n$. Then there exists a deformation $f: X \rightarrow D$ of $\left(X_{0}, x_{0}\right)$ such that $X_{t}, t \in D-\{0\}$, has exactly $p$ singular points $x_{1}, \ldots, x_{p}$ and $\left(X_{t}, x_{i}\right)$ is an ordinary $n_{i}$-tuple point. $(p=0$ is allowed; this means that $\left(X_{0}, x_{0}\right)$ is smoothable).

(3) The parameter space $S$ of the semiuniversal deformation of $\left(X_{0}, x_{0}\right)$ is of pure dimension $2 n-3$.

Proof. (1) By (4.2.2) we have $\mu_{0}-\mu_{t} \geqq \delta_{0}-\delta_{t}$ and by (1.2.4) $\mu_{0}=\delta_{0}, \mu_{t} \geqq \delta_{t}$. Therefore $\mu_{t}=\delta_{t}$ and (1) follows again by (1.2.4).

(2) Take any $\left(n_{1}-1\right)$ lines of $X_{0}$ and translate their common intersection along any other line $\ell$ (cf. Fig. 3).

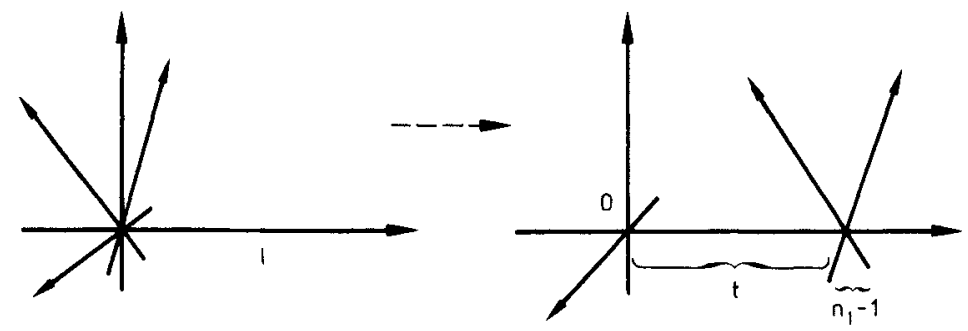

Fig. 3 
Of course this can be done by a deformation of the parametrization. But since $\mu$ stays constant, this indeed defines a deformation of $X_{0}$ (7.1.3). Now continue with the remaining lines through 0 by induction. Those lines through 0 which are left can be translated into ordinary double points and then smoothed (see also [Pi]).

(3) This is indeed a corollary of a result of P. Deligne. The dimension formula follows from a formula of Deligne, which can easily be applied to quasihomogeneous singularities (cf. $\left[\mathrm{G}_{2}\right]$ ).

Remark. One can show that the Zariski tangent space of $S$ has dimension $n(n-2)$ if $n \geqq 3$. Hence $S$ is smooth iff $n=1,2,3$ (cf. $\left[G_{2}\right]$ ).

The smooth fiber of the ordinary $n$-tuple point is a disc with $n-1$ holes. This follows since $\mu=n-1$ and since the fiber has $n$ boundary components (cf. Fig. 4).

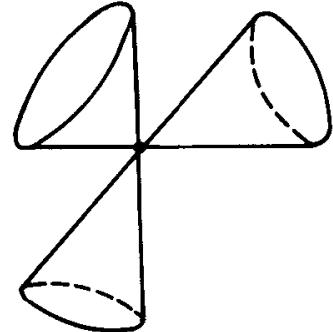

triple point

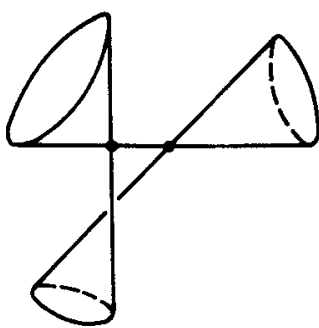

two double points

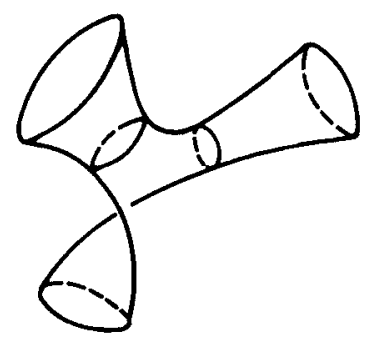

two vanishing cycles

Fig. 4

Let $S$ be the parameterspace of the semiuniversal deformation $\Phi: X \rightarrow S$ of the ordinary triple point. We know that it is smooth of dimension 3. Recall that the monodromy group is the image under the canonical representation

$$
\rho: \pi_{1}(S-D, t) \rightarrow \operatorname{Aut}\left(H_{1}\left(X_{t}, \mathbb{Z}\right)\right),
$$

where $D \subset S$ is the discriminant of $\Phi$ and $t \in S-D$ a base point. We claim

The monodromy group of the ordinary triple point is trivial. In particular, any geometric monodromy corresponding to an element $\gamma \in \pi_{1}(S-D, t)$ has fixed points.

Proof. By the local Lefschetz theorem, each element $\gamma \in \pi_{1}(S-D, t)$ can be represented by some loop in $S \cap L-\left\{p_{1}, \ldots, p_{q}\right\}$ where $L$ is a generic complex line in $\mathbb{C}^{3}$ near $0(0 \notin L, t \in L)$ which intersects $D$ in $p_{1}, \ldots, p_{q}$. By the above consideration, the generic fiber over $D$ has one ordinary double point, so $D$ is generically reduced and we may assume that the $p_{i}$ are simple points on $D$. Since $\gamma$ is the composition of simple loops around each point $p_{i}$ in the obvious way, we may assume that $\gamma$ is a simple loop around one point $p$ only and that the fiber $X_{p}$ contains exactly one ordinary double point. Denote by $e \in H_{1}\left(X_{t}, \mathbb{Z}\right)$ the vanishing cycle corresponding to this double point. By the Picard-Lefschetz formula we obtain

$$
\rho(\gamma)(\alpha)=\alpha-\langle\alpha, e\rangle e, \quad \alpha \in H_{1}\left(X_{t}, \mathbb{Z}\right),
$$

where $\langle$,$\rangle denotes the intersection pairing. But as we have seen above,$ $H_{1}\left(X_{t}, \mathbb{Z}\right)$ has a basis of non-intersecting cycles with self-intersection 0 , hence $\langle\alpha, e\rangle=0$ and we are done. 
Since $\mu=2$ and $X_{t}$ is connected, the Lefschetz number of $\rho(\gamma)$ is equal to -1 , hence each diffeomorphism of $X_{t}$ representing $\rho(\gamma)$ must have fixed points.

Lê Dũng Tràng ( $\left.\left[L \hat{e}_{2}\right]\right)$ has shown that for hypersurface singularities the classical monodromy on the homology can be represented by a characteristic diffeomorphism (=geometric monodromy) without fixed points. As A'Campo pointed out to us a similar result holds for complete intersections: there exists always a 1-parameter deformation and a corresponding local geometric monodromy which has no fixed points. More general and more precisely the following holds:

Theorem 7.2.7 (A'Campo). Let $(X, x)$ be a germ of a reduced complex space and $f:(X, x) \rightarrow(\mathbb{C}, 0)$ a deformation of $\left(X_{0}, x\right)=\left(f^{-1}(0), x\right)$. Assume $X-X_{0}$ to be smooth and $f \in \mathrm{m}^{2}$ where $\mathrm{m}$ is the maximal ideal of $(X, x)$. Then the local monodromy of $f$ can be represented by a geometric monodromy without fixed points.

The proof follows from $\left[\mathrm{A}^{\prime} \mathrm{C}\right]$, théorème 5 , after resolving the singularities of $f$ via a morphism $\pi: \tilde{X} \rightarrow X$ such that $\tilde{X}$ is smooth, $(f \circ \pi)^{-1}(0)$ consists of smooth components which intersect normally and $X-(f \circ \pi)^{-1}(0) \cong X-X_{0}$. Note that the assumption $f \in \mathrm{m}^{2}$ implies that $f \circ \pi$ vanishes on $\pi^{-1}(0)$ at least of order 2.

Remark 7.2.8. We note that the ordinary $n$-tuple point is a simple ( 0 -modular) singularity in the sense of Arnold. For the ordinary triple point the discriminant $D \subset S$ consists of the 3 coordinate hyperplanes. Therefore $S-D$ is an EilenbergMcLane space - supporting a conjecture of R. Thom. We do not know whether this is true also for $n \geqq 4$.

\section{References}

[A'C] A'Campo, N.: La fonction zêta d'une monodromie, Comment. Math. Helv. 50, 233-248 (1975)

[A-K] Altman, A., Kleiman, S.: Introduction to Grothendieck Duality Theory, SLN 146, Berlin Heidelberg New York: Springer-Verlag 1970

[Ba] Bassein, R.: On Smoothable Curve Singularities: Local Methods, Math. Ann 230, 273-277 (1977)

[Be] Berger, R.: Differentialmoduln eindimensionaler lokaler Ringe, Math. Zeitschrift 81, 326354 (1963)

[B-G] Buchweitz, R.-O., Greuel, G.-M.: Le nombre de Milnor, équisingularité et deformations de singularités des courbes réduites, C. R. Acad. Sci. Paris, 288, 35-38 (1979)

[B-G-G]Briancon, J., Galligo, A., Granger, M.: Déformations équisingulières des germes de courbes gauches réduites, preprint of the Dep. of Math., University of Nice, France

[Br] Brieskorn, E.: Die Monodromie der isolierten Singularitäten von Hyperflächen, manuscripta math. 2, 103-161 (1970)

[Do] Douady, A.: Séminaire Bourbaki, Exp. 404, 1971/1972; see also: Douady, A., Verdier, J.L., Séminaire de l'E.N.S. 1971/1972, astérisque 16 (1974)

[EGA] Grothendieck, A., Dieudonné, J.: Eléments de géométrie algébrique I-IV, Publ. de l'IHES $4,8,11,17,20,24,28,32$

[El] Elzein, F.: Complexe dualisant et applications, Thèse Paris VII, 1976

[Gi] Giusti, M.: Classification des singularités isolées simples d'intersection complètes, preprint

[Go] Godement, R.: Théorie des faisceaux, Paris: Hermann 1964

$\left[\mathrm{G}_{1}\right]$ Greuel, G.-M.: Der Gauss-Manin-Zusammenhang isolierter Singularitäten von vollständigen Durchschnitten, Math. Ann. 214, 235-266 (1975) 
$\left[\mathrm{G}_{2}\right]$ Greuel, G.-M.: Deformation spezieller Kurvensingularitäten und eine Formel von Deligne, preprint

[Gr] Grothendieck, A.: Local Cohomology, SLN 41, Berlin Heidelberg New York: SpringerVerlag 1967

[Ha] Hamm, H.: Habilitationsschrift, Göttingen 1974

[H] Hartshorne, R.: Residues and Duality, SLN 20, Berlin Heidelberg New York: SpringerVerlag 1966

[H-K ${ }_{1}$ ] Herzog, J., Kunz, E.: Der kanonische Modul eines Cohen-Macaulay-Rings, SLN 238, Berlin Heidelberg New York: Springer-Verlag 1971

[H-K $\left.{ }_{2}\right]$ Herzog, J., Kunz, E.: Die Werthalbgruppe eines lokalen Rings der Dimension 1, Ber. Heidelberger Akad. Wiss., II. Abhandlung 1971

[Hi] Hironaka, H.: On the Arithmetic Genera and the Effective Genera of Algebraic Curves, Mem. Coll. Sci., Univ. of Kyoto 30, 177-195 (1957)

[Hu] Hudson, J.F.P.: Piecewise linear topology, W.A. Benjamin 1969

[K-V] Kiehl, R., Verdier, J.L.: Ein einfacher Beweis des Kohärenzsatzes von Grauert, Math. Ann. 195, 24-50 (1971)

[Lê, $]$ Lê, D.T.: Sur un critère d'equisingularité, in: Séminaire F. Norguet, Proceedings 19701973. SLN 409, Berlin Heidelberg New York: Springer-Verlag 1974

$\left[\mathrm{Le}_{2}\right] \quad$ Lê, D.T.: La monodromie n'a pas de points fixes, J. of Fac. Sc. Univ. Tokio, 22, 409-427 (1975)

[L-L-T] Lejeune, M., Lê, D.T., Teissier. B.: Sur un critère d'équisingularité, C. R. Acad. Paris 271, 1065-1067, Série A (1970)

[L-R] Lê, D.T., Ramanujam, C.P.: The Invariance of Milnor's Number implies the Invariance of the Topological Type, Am. J. of Math., 98, 67-78 (1976)

[Li] Lickorish, W.B.R.: The Piecewise Linear Unknotting of Cones, Topology 4, 67-91 (1965)

[L-T] Lipman, J., Teissier, B.: Introduction to volume IV of the Collected Papers of $\mathrm{O}$. Zariski, in press (1980)

[Mi] Milnor, J.: Singular Points of Complex Hypersurfaces, Ann. of Math. Studies 61, Princeton 1968

[Pi] Pinkham, H.: Deformations of Algebraic Varieties with $\mathbb{G}_{m !}$-action, astérisque 20, 1974

[Se] Serre, J-P.: Groupes algébriques et corps des classes, Paris: Hermann 1959

[ $\left.\mathrm{Te}_{1}\right]$ Teissier, B.: Introduction to Equisingularity Problems, Proc. of the AMS 29, 593-632 (1975)

$\left[\mathrm{Te}_{2}\right]$ Teissier, B.: The Hunting of Invariants in the Geometry of Discriminants. In: Real and Complex Singularities (P. Holme, ed.), Northholland 1978

[ $\left.\mathrm{Te}_{3}\right]$ Teissier, B.: Résolution simultanée I, II. In: Séminaire Demazure-Pinkham-Teissier 1976/1977, in press; see also: Teissier, B.: Sur divers conditions numerique d'équisingularité des familles de courbes, preprint at the Ecole Polytechnique, EP/M 208.0675, 1975

$\left[\mathrm{Te}_{4}\right]$ Teissier, B.: Déformations à type topologique constant, in: Séminaire Douady-Verdier 1971/1972, astérisque 16, 1974

[Ti] Timourian, J.G.: The Invariance of Milnor's Number implies Topological Triviality, Am. J. of Math. 99, 437-466 (1977)

$\left[Z_{1}\right] \quad$ Zariski, O.: Studies in Equisingularity, I, II, III, Am. J. of Math. 87, 507-536, 972-1006 (1965) Am. J. of Math. 90. 961-1023 (1968)

$\left[Z_{2}\right] \quad$ Zariski, O.: Open Questions in the Theory of Singularities, Bull. AMS 77, 481-491 (1971)

$\left[Z_{3}\right] \quad Z a r i s k i, O .:$ The Elusive Concept of Equisingularity and Related Questions. In: Algebraic Geometry, The Johns Hopkins Centennial Lectures (J.I. Igusa, ed.), Johns Hopkins University Press 1977

$\left[Z_{4}\right] \quad$ Zariski, O.: Characterization of Plane Algebroid Curves whose Module of Differentials has Maximum Torsion, Proc. National Academy of Sciences, 56, 781-786 (1966)

$\left[\mathrm{Z}_{5}\right]$ Zariski, O.: Contributions to the Problem of Equisingularity. In: Problems in Algebraic Geometry, C.I.M.E., Ed. Cremonese, Roma, 1969 